\title{
Spectral-infinite-element simulations of gravity anomalies
}

\author{
Hom Nath Gharti, ${ }^{1}$ Jeroen Tromp ${ }^{1,2}$ and Stefano Zampini ${ }^{3}$ \\ ${ }^{1}$ Department of Geosciences, Princeton University, Princeton, New Jersey, USA. E-mail: hgharti@princeton.edu \\ ${ }^{2}$ Program in Applied \& Computational Mathematics, Princeton University, Princeton, New Jersey, USA \\ ${ }^{3}$ Extreme Computing Research Center Computer, Electrical and Mathematical Sciences and Engineering Division, King Abdullah University of Science and \\ Technology, Thuwal, Saudi Arabia
}

Accepted 2018 August 3. Received 2018 July 29; in original form 2018 February 02

\begin{abstract}
S UMMAR Y
Gravity anomalies induced by density heterogeneities are governed by Poisson's equation. Most existing methods for modelling such anomalies rely on its integral solution. In this approach, for each observation point, an integral over the entire density distribution needs to be carried out, and the computational cost is proportional to the number of observation points. Frequently, such methods are sensitive to high density contrasts due to inaccurate resolution of the volume integral. We introduce a new approach which directly solves a discretized form of the Poisson/Laplace equation. The main challenge in our approach involves the unbounded nature of the problem, because the potential exists in all of space. To circumvent this challenge, we combine a mapped infinite-element approach with a spectral-element method. Spectral elements represent the domain of interest, and a single layer of infinite elements captures outer space. To solve the weak form of the Poisson/Laplace equation, we use Gauss-LegendreLobatto (GLL) quadrature in spectral elements inside the domain of interest. Outside the domain, we use Gauss-Radau quadrature in the infinite direction, and GLL quadrature in the other directions. We illustrate the efficiency and accuracy of our method by comparing calculated gravity anomalies for various density heterogeneities with corresponding analytical solutions. Finally, we consider a complex 3-D model of an ore mine, which consists of both positive and negative density anomalies.
\end{abstract}

Key words: Gravity anomaly; Poisson's equation; Unbounded domain; Spectral-infiniteelement method.

\section{INTRODUCTION}

Mass density heterogeneities produce gravity anomalies, which can be measured on Earth's surface or in space. The gravitational potential is governed by Poisson's equation inside the density distribution, and by Laplace's equation elsewhere. The solution to Poisson's equation may be expressed as an integral over the mass distribution, and most existing methods for computing gravity are based on direct manipulation of this integral solution. If the gravity anomaly needs to be computed inside the density anomaly, special procedures are necessary to avoid singularities.

There have been studies of gravity anomalies induced by 2-D objects with a uniform density anomaly (Talwani et al. 1959; Bott 1960; Won \& Bevis 1987), 3-D objects with a uniform density anomaly (Talwani \& Ewing 1960; Nagy 1966; Plouff 1976; Banerjee \& Das Gupta 1977), spherical and ellipsoidal objects with a 1-D density anomaly (Johnson \& Litehiser 1972), homogeneous polyhedrons (Werner 1994) as well as 3-D objects with variable density anomalies (Chakravarthi et al. 2002; García-Abdeslem 2005). The software package GBOX is widely used for computing gravity anomalies due to uniform rectangular prisms (Blakely 1995). It implements the analytical solution for a rectangular prism first given by Plouff (1976). GBOX can also be used to compute gravity anomalies due to irregular objects by subdividing the object into a number of uniform rectangular subprisms and superposing their contributions. In the context of the coupled elastic-gravitational equations, Poisson's equation is generally solved using a spherical harmonics normal-mode approach (Pollitz 1997; Spada et al. 2004; Al-Attar \& Woodhouse 2008).

Because the integrand in the integral solution is dependent on the observation point, a volume integral has to be carried out for each observation point. Therefore, compute time is proportional to the number of observation points. For a large number of observation points and a large density anomaly, the computational cost may be excessive. The accuracy and efficiency of these methods may be significantly improved with numerical quadrature. For example, Martin et al. (2017) used spectral-elements to discretize the model and GLL quadrature for volume integration. 
Very few methods are based on solving the weak form of Poisson's equation. In this context, the main challenge is the infinite boundary condition. Cai \& Wang (2005) used a finite-element method with approximate boundary conditions to compute the gravitational potential on the outer surface of an extended model, under the assumption that the gravitational potential is of order $r^{-4}$ at a distance $r$ from the centre of mass. For global seismic wave propagation simulation, Chaljub \& Valette (2004) solved the Poisson's equation using spectral-element method first approximating the Neumann boundary conditions on the free surface using spherical harmonics. An alternative approach is to consider a very large domain that includes portions of outer space. This strategy requires large computational resources and is often inaccurate (Tsynkov 1998). A higher-order solver based on a convolution integral was also proposed to solve the unbounded Poisson equation (Hejlesen et al. 2013). Alternatively, one may use spherical harmonics for spherical density anomalies (Dahlen 1974; Tromp \& Mitrovica 1999).

In solid and fluid mechanics, the displacement descent approach (Bettess 1977; Medina \& Taylor 1983; El-Esnawy et al. 1995) and the coordinate ascent approach (Beer \& Meek 1981; Zienkiewicz et al. 1983; Kumar 1985; Angelov 1991) are both widely used to solve unbounded problems. In the displacement descent approach, an element in the physical domain is mapped to an element in a natural domain of interval $[0, \infty]$. This is achieved by multiplying the standard interpolation functions by suitable decay functions. Since the integration interval is $[0, \infty]$, classical Gauss-Legendre quadrature cannot be employed. Either Gauss-Legendre quadrature has to be modified to accommodate the $[0, \infty]$ interval, or Gauss-Laguerre quadrature can be used (Mavriplis 1989). In terms of programming, both the Jacobian of the mapping and the numerical quadrature have to be modified from the classical finite-element method. The coordinate ascent approach is also referred to as the 'mapped infinite element' approach. In this technique, an element that extends to infinity in the physical domain is mapped to a standard natural element with interval $[-1,1]$. This is achieved by defining shape functions using a point outside the element opposite to the infinite direction; this point is frequently called a pole. The corresponding shape function possesses a singularity at the far end of the infinite element to map the infinite location. Standard Gauss-Legendre quadrature can be used. In terms of programming, only the Jacobian of the mapping needs to be modified, and quadrature identical to the classical finite-element method can be used.

The spectral-element method (SEM) is a high-order finite element method which uses nodal quadrature, specifically, Gauss-LegendreLobatto (GLL) quadrature. For dynamic problems, the mass matrix is diagonal by construction. The method is highly accurate and efficient, and is widely used in applications involving wave propagation (Faccioli et al. 1997; Seriani \& Oliveira 2008; Tromp et al. 2008; Peter et al. 2011), fluid dynamics (Patera 1984; Canuto et al. 1988; Deville et al. 2002), as well as for quasistatic problems (Gharti et al. 2012a,b).

To solve the unbounded Poisson/Laplace equation efficiently, we combine the infinite-element approach based on coordinate ascent with the SEM. From hereon, we refer to this method as a 'spectral-infinite-element method (SIEM)'. Gharti \& Tromp (2017) previously developed the SIEM to compute background gravity on a global scale. This paper details the development and implementation of the SIEM to compute gravity anomalies. The method is validated based on calculations of the gravity anomaly for a range of problems. Finally, we demonstrate an application of the method to an ore mine in Finland.

\section{SPECTRAL-INFINITE-ELEMENT FORMULATION}

\subsection{Governing equation}

In this paper, we are concerned with perturbations in the gravitational potential, $\delta \Phi$, induced by perturbations in mass density, $\delta \rho$. Since the Laplacian, $\nabla^{2}$, is a linear operator, Poisson's equation may be used to calculate gravitational perturbations induced by such density perturbations (see e.g. Dahlen \& Tromp 1998), that is

$\nabla^{2} \delta \Phi=4 \pi G \delta \rho$

where $G$ denotes the universal gravitational constant. Let $\Omega$ denote the domain of interest with boundary $\Gamma$, and let $\delta \Omega$ denote the region with a non-zero density perturbation, as illustrated in Fig. 1 . All of space is denoted by $\bigcirc$, and the 'boundary' at infinity is denoted by $\Gamma_{\infty}$. Outside the density anomaly domain $\delta \Omega$, the mass density anomaly vanishes, and the governing eq. (1) reduces to Laplace's equation: $\nabla^{2} \delta \Phi$ $=0$. The associated boundary conditions are

$$
\begin{aligned}
{[\delta \Phi]_{-}^{+}=0 } & \text { on } \quad \Gamma, \\
{[\hat{\mathbf{n}} \cdot \nabla \delta \Phi]_{-}^{+}=0 } & \text { on } \quad \Gamma,
\end{aligned}
$$

where $[\cdot]_{-}^{+}$denotes a jump in the enclosed quantity when going from the - side of boundary $\Gamma$ to the + side, and where $\hat{\mathbf{n}}$ denotes the unit outward normal to the boundary, pointing from the - side to the + side. If the model domain $\Omega$ has internal discontinuities - as is the case at phase boundaries in the Earth's interior-the gravitational field satisfies boundary conditions (2) across each discontinuity. At $\Gamma_{\infty}$, the field satisfies the boundary condition

$\delta \Phi=0 \quad$ on $\Gamma_{\infty}$. 


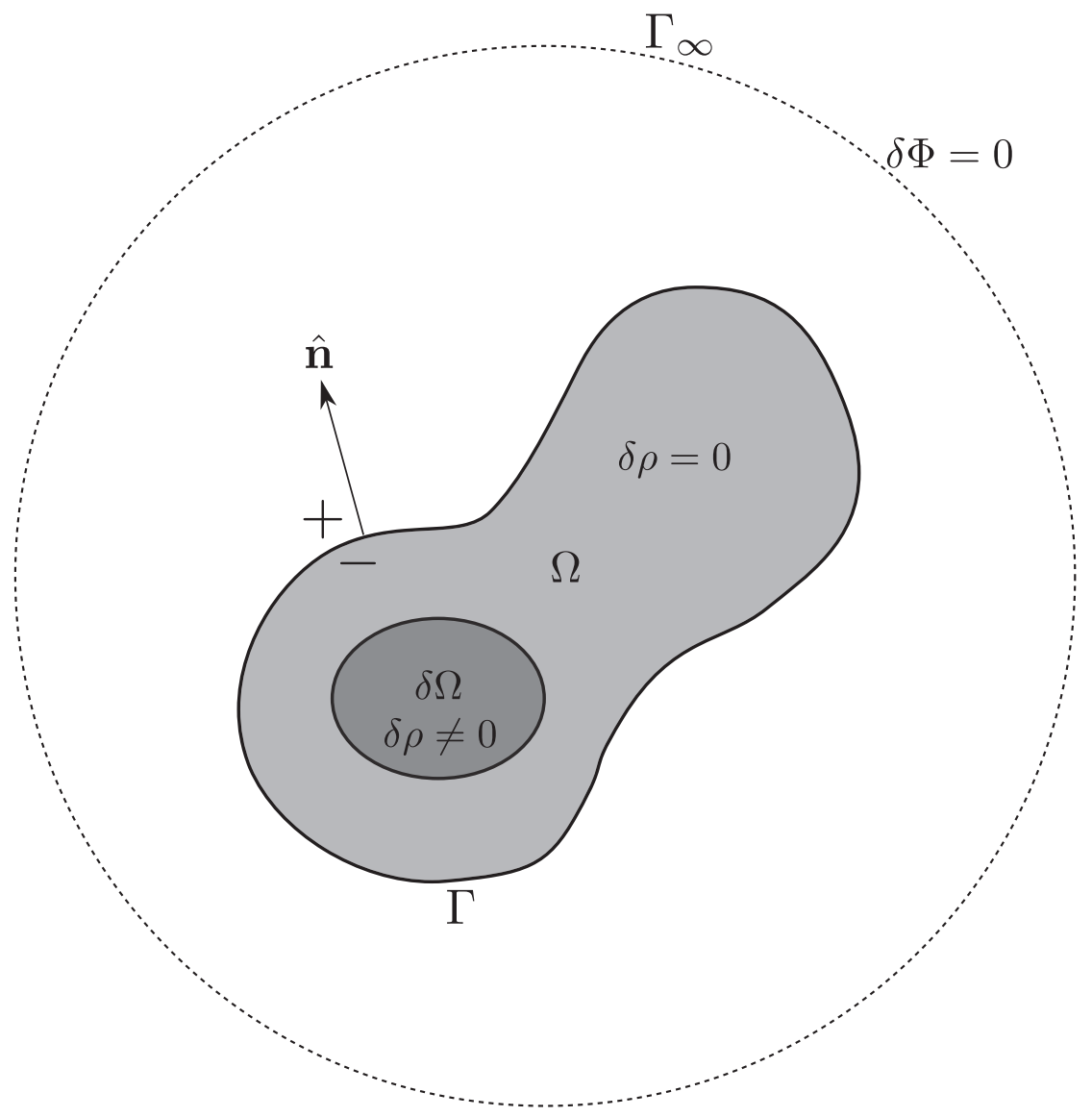

Figure 1. Schematic diagram of an unbounded domain with a fictitious boundary $\Gamma_{\infty}$ on which the perturbed gravitational potential vanishes, $\delta \Phi=0$. The domain contains a region of interest, $\Omega$, without density perturbations, $\delta \rho=0$, and a region $\delta \Omega$, with non-zero density anomalies, $\delta \rho \neq 0$. The domain $\Omega$ has a boundary $\Gamma$ with unit outward normal $\hat{\mathbf{n}}$, pointing from the inside of the domain $(-)$ to the outside of the domain $(+)$.

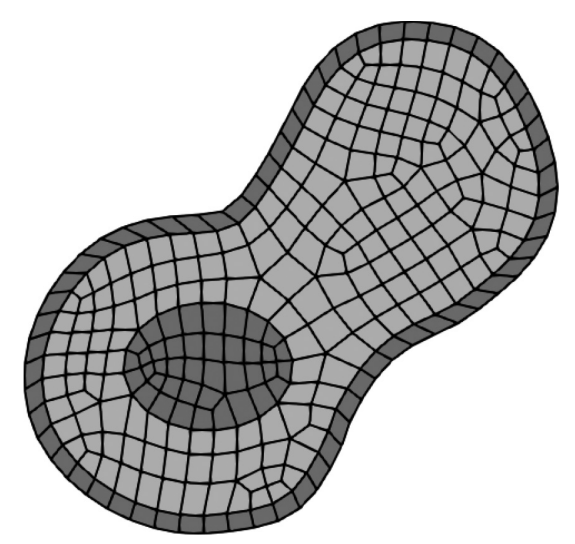

Figure 2. The domain is discretized using spectral elements. The mesh honors the density anomaly (inner dark grey elements). A single layer of infinite elements is added outside the domain (outer dark grey elements).

\subsection{Discretization}

The weak form of the governing eq. (1) subject to boundary conditions (2) and (3) is

$\int_{\bigcirc} \nabla w \cdot \nabla \delta \Phi \mathrm{d} V=-4 \pi G \int_{\delta \Omega} w \delta \rho \mathrm{d} V+\int_{\Gamma_{\infty}} w \hat{\mathbf{n}} \cdot \nabla \delta \Phi \mathrm{d} S$

where $w$ denotes a test function, and where we have used the fact that the mass density anomaly vanishes outside of $\delta \Omega$.

The domain $\Omega$ is meshed using spectral elements (Fig. 2). A single layer of infinite elements is added outside the domain in order to reproduce the behaviour of outer space. As we discuss in more detail later, spectral and infinite elements share the same interpolation functions, namely Lagrange polynomials, but use different quadrature. Thus, the gravitational potential perturbation $\delta \Phi$ is discretized in 
natural coordinates $\xi$ as

$\delta \Phi(\xi)=\sum_{\alpha=1}^{n} \delta \Phi_{\alpha} N_{\alpha}(\xi)$,

where $\delta \Phi_{\alpha}$ denotes the field value at quadrature point $\xi_{\alpha}$, and $N_{\alpha}$ denotes an interpolation function. The total number of quadrature points in an element is $n$, and is given by the product of the number of quadrature points in each dimension, $n^{j}, j=1,2,3$, that is, $n=\prod_{j=1}^{3} n^{j}$. The interpolation functions $N_{\alpha}$ in natural coordinates are determined by a tensor product of one-dimensional Lagrange polynomials, that is

$N_{\alpha^{j}}^{j}\left(\xi^{j}\right)=\prod_{\substack{\beta=1 \\ \beta \neq \alpha^{j}}}^{n^{j}} \frac{\left(\xi^{j}-\xi_{\beta}^{j}\right)}{\left(\xi_{\alpha^{j}}^{j}-\xi_{\beta}^{j}\right)}$,

such that

$N_{\alpha}(\xi)=\prod_{j=1}^{3} N_{\alpha^{j}}^{j}\left(\xi^{j}\right)$.

Here $\alpha$ denotes the index of quadrature point $\xi_{\alpha}=\left\{\xi_{\alpha^{1}}, \xi_{\alpha^{2}}, \xi_{\alpha^{3}}\right\}$.

The test function $w$ is taken to be an interpolation function $N_{\alpha}$, making the approach a Galerkin method. Upon substituting $w=N_{\alpha}$ and $\delta \Phi$ given by eq. (5) in eq. (4), we obtain a set of elemental linear equations that may be written conveniently in matrix-vector form:

$\mathbf{K}_{e} \delta \boldsymbol{\Phi}_{e}=\mathbf{F}_{e}$.

The quantities $\mathbf{K}_{e}$ and $\mathbf{F}_{e}$ are known, respectively, as the stiffness matrix and force vector of an element. Similarly, $\delta \boldsymbol{\Phi}_{e}$ is the gravitational potential vector. Symbolically, we write

$$
\begin{aligned}
\mathbf{K}_{e} & =\int_{\Omega_{e}} \mathbf{B}^{T} \mathbf{B} \mathrm{d} V, \\
\mathbf{F}_{e} & =-4 \pi G \int_{\delta \Omega_{e}} \delta \rho \mathbf{N}_{e} \mathrm{~d} V+\int_{\Gamma_{\infty}}(\hat{\mathbf{n}} \cdot \nabla \delta \Phi)_{e} \mathbf{N}_{e} \mathrm{~d} S,
\end{aligned}
$$

where

$\mathbf{B}=\mathbf{D} \mathbf{N}_{e}^{T}$,

such that

$\mathbf{D}=\left\{\begin{array}{lll}\frac{\partial}{\partial x} & \frac{\partial}{\partial y} & \frac{\partial}{\partial z}\end{array}\right\}^{T}$,

and

$\mathbf{N}_{e}=\left\{\begin{array}{lll}N_{1} & N_{2} & N_{3} \cdots N_{n}\end{array}\right\}^{T}$,

$\delta \boldsymbol{\Phi}_{e}=\left\{\delta \Phi_{1} \quad \delta \Phi_{2} \quad \delta \Phi_{3} \cdots \delta \Phi_{n}\right\}^{T}$.

After assembling the elemental matrices and vectors, we obtain a set of global linear equations

$\mathbf{K} \delta \boldsymbol{\Phi}=\mathbf{F}$,

where $\mathbf{K}$ and $\mathbf{F}$ are known, respectively, as the global stiffness matrix and global force vector. Similarly, $\delta \boldsymbol{\Phi}$ is the global gravitational potential vector.

On the fictitious boundary $\Gamma_{\infty}$, both the gravity anomaly and its gradient vanish, but in the numerical solution we cannot prescribe both conditions simultaneously on the same boundary. Therefore, we impose a zero gravity anomaly, $\delta \Phi=0$, and regard its gradient, $\nabla \delta \Phi$, as unknown on the boundary $\Gamma_{\infty}$, such that the surface integral $\int_{\Gamma_{\infty}} w \hat{\mathbf{n}} \cdot \nabla \delta \Phi \mathrm{d} S$ is unknown. We call the corresponding unknown part of the global force vector $\mathbf{F}_{1}$, and its known part, determined by $-4 \pi G \int_{\delta \Omega} w \delta \rho \mathrm{d} V, \hat{\mathbf{F}}_{2}$. We note that if the gradient is computed on $\Gamma_{\infty}$ based on the numerical solution, it is zero within machine precision. Similarly, the global potential vector, $\delta \boldsymbol{\Phi}$, is split in terms of a known component, $\delta \hat{\boldsymbol{\Phi}}_{1}$, which corresponds to values on $\Gamma_{\infty}$ which are zero, and an unknown component, $\delta \boldsymbol{\Phi}_{2}$, which corresponds to the rest of the domain. Thus, we may partition the global equation as follows:

$$
\left[\begin{array}{ll}
\mathbf{K}_{11} & \mathbf{K}_{12} \\
\mathbf{K}_{21} & \mathbf{K}_{22}
\end{array}\right]\left[\begin{array}{l}
\delta \hat{\boldsymbol{\Phi}}_{1} \\
\delta \boldsymbol{\Phi}_{2}
\end{array}\right]=\left[\begin{array}{c}
\mathbf{F}_{1} \\
\hat{\mathbf{F}}_{2}
\end{array}\right],
$$

resulting in two sets of linear equations, namely,

$\mathbf{K}_{11} \delta \hat{\boldsymbol{\Phi}}_{1}+\mathbf{K}_{12} \delta \boldsymbol{\Phi}_{2}=\mathbf{F}_{1}$. 

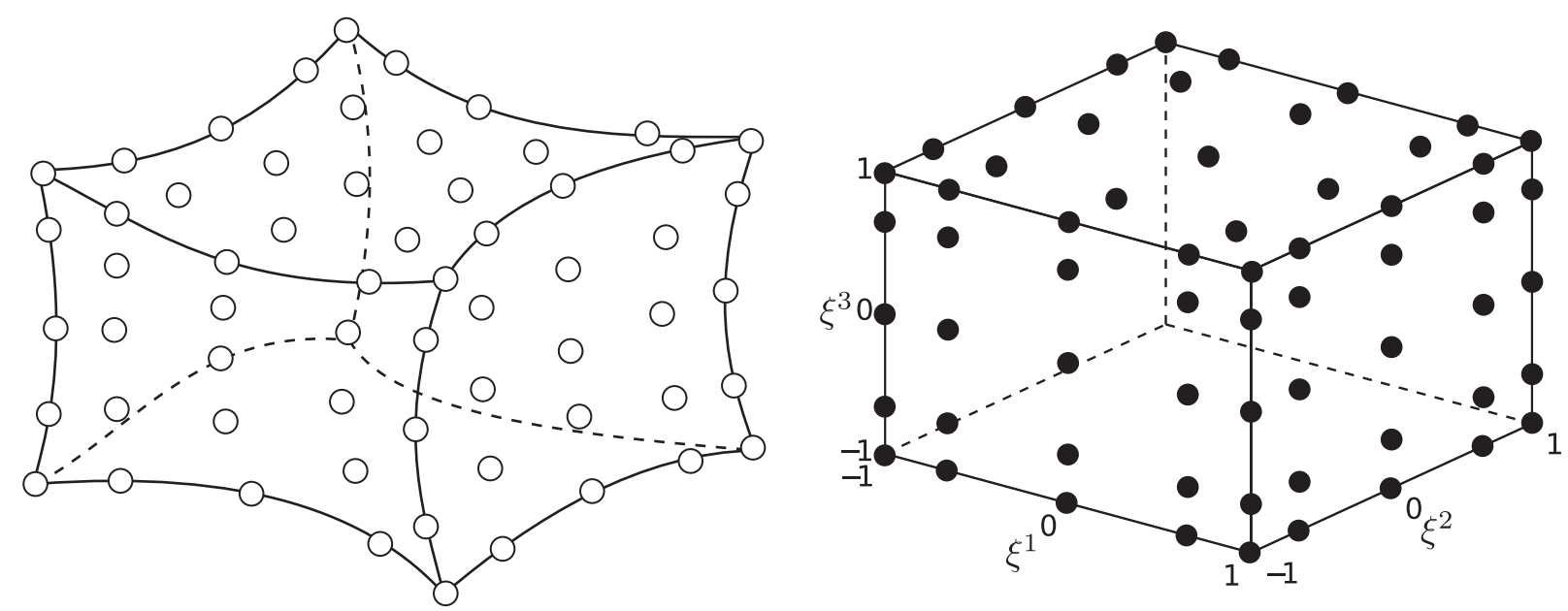

Figure 3. Left-hand panel: a typical spectral element with five interpolation nodes in each dimension (open circles). Right-hand panel: the same spectral element mapped to its natural coordinates. Gauss-Legendre-Lobatto points (solid black circles) are used for numerical integration. Integration points and interpolation nodes coincide. Only interpolation nodes on the three visible faces are shown for clarity.

and

$\mathbf{K}_{21} \delta \hat{\boldsymbol{\Phi}}_{1}+\mathbf{K}_{22} \delta \boldsymbol{\Phi}_{2}=\hat{\mathbf{F}}_{2}$,

First, the unknown part, $\delta \boldsymbol{\Phi}_{2}$, is obtained by noting that $\delta \hat{\boldsymbol{\Phi}}_{1}=0$ and rewriting eq. (17) as

$\mathbf{K}_{22} \delta \boldsymbol{\Phi}_{2}=\hat{\mathbf{F}}_{2}$,

leading to a system of linear equations for $\delta \boldsymbol{\Phi}_{2}$. Once $\delta \boldsymbol{\Phi}_{2}$ is determined, $\mathbf{F}_{1}$ can be directly obtained from eq. (16).

After determining the gravity potential $\delta \Phi$, we compute the perturbed gravitational acceleration, $\delta \mathbf{g}$, by simply calculating a numerical derivative within a spectral element:

$\delta \mathbf{g}=-\nabla \delta \Phi=-\sum_{\alpha=1}^{n} \delta \Phi_{\alpha} \mathbf{J}_{\alpha}^{-1} \cdot \nabla N_{\alpha}$,

where $\mathbf{J}_{\alpha}^{-1}$ denotes the inverse of the Jacobian of the mapping (see Section 2.3) at node $\alpha$. The vertical component of the perturbed gravity acceleration is often referred to as the surface gravity anomaly. One key advantage of the spectral-element method is that derivatives are directly computed on quadrature points, rendering this operation highly accurate. However, derivatives may be discontinuous across elemental boundaries, and therefore on such boundaries we average over all elements that touch a given node.

\subsection{Mapping}

For numerical integration, a point $\mathbf{x}=\left\{x^{i}\right\}$ in a physical element is mapped to a point $\xi=\left\{\xi^{j}\right\}$ in the natural element, as illustrated in Fig. 3 . This mapping is different for the spectral-element and infinite-element domains, as described below.

\subsubsection{Spectral elements}

A spectral element is mapped to a natural element using the transformation

$\mathbf{x}(\boldsymbol{\xi})=\sum_{\alpha=1}^{n_{\mathrm{g}}} \mathbf{x}_{\alpha} M_{\alpha}(\boldsymbol{\xi})$.

Here $M_{\alpha}$ denotes a shape function and $n_{\mathrm{g}}$ the number of geometrical nodes, $\mathbf{x}_{\alpha}$, of an element. The shape function $M_{\alpha}$ is defined similar to the interpolation function (eq. 5). However, the number of interpolation points, $n$, and the number of geometrical points, $n_{g}$, may differ. In general, $n_{g}<n$ for the spectral-element method, leading to a subparametric formulation. The Jacobian matrix of the transformation is determined using the relation $J^{i j}(\boldsymbol{\xi})=\partial x^{i}(\boldsymbol{\xi}) / \partial \xi^{j}$. We use GLL quadrature for numerical integration, in which the interpolation and quadrature points are identical.

\subsubsection{Infinite elements}

Inside the domain $\Omega$, geometrical nodes are used to map an element from the physical domain to the natural domain (Fig. 4, left-hand panel). 

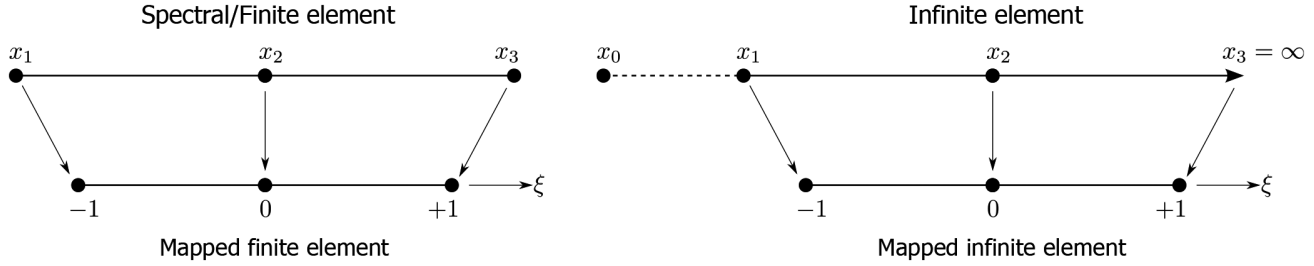

Figure 4. Mapping of a 1-D element to natural coordinates. The element consists of three geometrical nodes. Left-hand panel: spectral/finite element. Right-hand panel: infinite element.
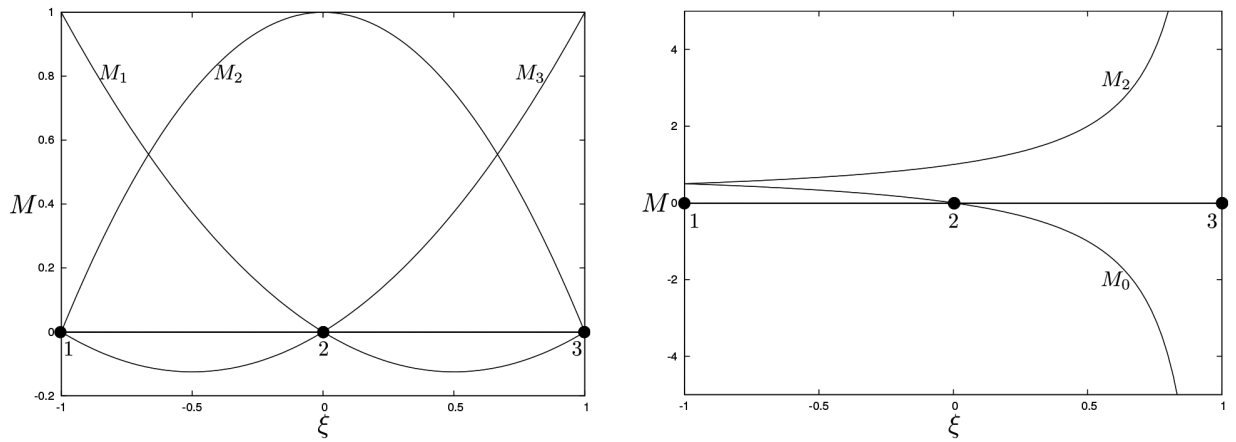

Figure 5. Shape functions for a 1-D element with three geometrical nodes: 1, 2 and 3. Left-hand panel: spectral/finite element. Right-hand panel: infinite element.

Outside the domain $\Omega$, we introduce a single layer of elements in which the gravity field is discretized using infinite elements (Fig. 2). For simplicity and clarity, we illustrate a 1-D mapping. For the infinite element shown in the Fig. 4(right-hand panel), the point $x_{0}$ is defined as the 'pole'. For a given pole, $x_{0}$, and node $x_{1}$, the intermediate node $x_{2}$ is determined by the relation $\gamma=\left(x_{2}-x_{0}\right) /\left(x_{1}-x_{0}\right)$. Generally, we set $\gamma=2$, so that point $x_{1}$ is located midway between points $x_{0}$ and $x_{2}$. The pole $x_{0}$ and node $x_{2}$ are used to map the element from the physical domain to the natural domain (Fig. 4, right-hand panel) using the transformation (Curnier 1983; Zienkiewicz et al. 1983)

$x=M_{0}(\xi) x_{0}+M_{2}(\xi) x_{2}$,

where the shape functions $M_{0}(\xi)$ and $M_{2}(\xi)$ are defined as

$M_{0}(\xi)=\frac{\xi}{\xi-1}$,
$M_{2}(\xi)=1+\frac{\xi}{1-\xi}$.

The shape functions $M_{0}(\xi)$ and $M_{2}(\xi)$ satisfy the relation

$M_{0}(\xi)+M_{2}(\xi)=1$.

These shape functions map an element in the physical domain, which is extended to infinity, to an element in the natural domain in the following manner (Fig. 4):

$\xi=-1 \rightarrow x=x_{1}$,

$\xi=0 \quad \rightarrow \quad x=x_{2}$,

$\xi=+1 \quad \rightarrow \quad x=x_{3}=\infty$.

For infinite elements, shape functions therefore become singular on right-end boundary nodes. Shape functions for regular spectral and infinite elements are plotted in Fig. 5.

To understand the implications of using infinite-element shape functions, we may express the field variable as a piecewise polynomial:

$\delta \Phi(\xi)=a_{0}+a_{1} \xi+a_{2} \xi^{2}+a_{3} \xi^{3}+\cdots$,

where, $a_{0}, a_{1}, a_{2}$, are constants. From eqs (21) and (22) we determine the inverse map

$\xi=1-\frac{\gamma\left(x_{1}-x_{0}\right)}{r}$,

where $r=x-x_{0}$ is the distance measured from the pole.

Substituting $\xi$ in eq. (25), we obtain

$\delta \Phi(r)=b_{0}+\frac{b_{1}}{r}+\frac{b_{2}}{r^{2}}+\frac{b_{3}}{r^{3}}+\cdots$, 

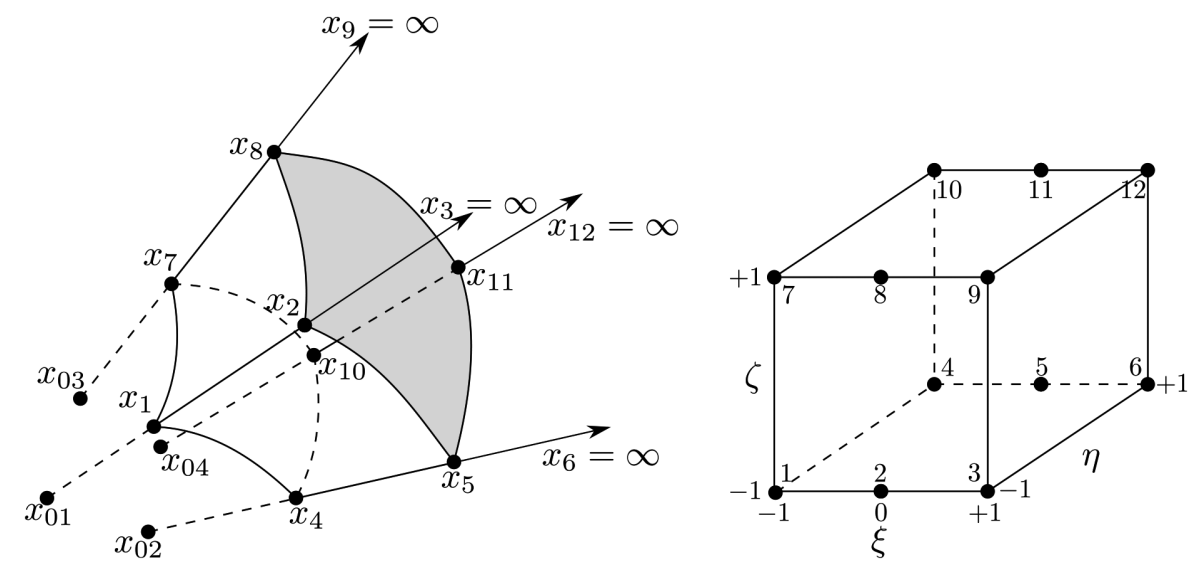

Figure 6. Left-hand panel: a general 3-D infinite element. Points $x_{01}, x_{02}, x_{03}$ and $x_{04}$ are the poles. Right-hand panel: 3-D infinite element mapped to its natural coordinates.

where $b_{0}, b_{1}, b_{2}$, are constants for given values of $x_{0}, x_{1} x_{2}$. Hence $\delta \Phi$ decays to $b_{0}$ when $r$ tends to $\infty$. For our problem, since the gravity anomaly decays to zero at $\infty$, the value of $b_{0}$ is zero. For solid mechanics problems, the pole is generally defined to be the point from which the 'disturbance' originates (Zienkiewicz et al. 2005). By analogy, we generally define the centre of mass of the density anomaly, $\overline{\mathbf{x}}$, as the pole, that is,

$\overline{\mathbf{x}}=\frac{\int_{V} \mathbf{x} \delta \rho(\mathbf{x}) \mathrm{d} V}{\int_{V} \delta \rho(\mathbf{x}) \mathrm{d} V}$.

However, we can set one or more poles, depending on the density anomaly. All poles have to be located opposite to the decay direction and outside the infinite element. The accuracy of the infinite-element approximation may be improved by increasing the order of the interpolation functions, but the shape functions remain the same. Alternatively, the shape functions for the infinite element can be defined using nodes 1 and 2 shown in Fig. 5 (Marques \& Owen 1984; Kumar 1985). Similarly, shape functions for particular decay functions, such as exponential or logarithmic decay, can also be derived (Abdel-Fattah et al. 2000). A typical mapping of a 3-D infinite element in a general physical domain to an element in the natural domain is shown in Fig. 6.

\subsection{Numerical integration}

In a spectral element, we use GLL quadrature:

$\int_{-1}^{1} f(\xi) \mathrm{d} \xi=w_{1} f(-1)+\sum_{\alpha=2}^{n-1} w_{\alpha} f\left(\xi_{\alpha}\right)+w_{n} f(1)$

where $f$ is a general function, $w_{\alpha}$ are the GLL weights of integration, and $\xi_{\alpha}$ are the quadrature points. GLL quadrature is exact for polynomials up to order $2 n-3$. Since the quadrature includes the end points of the interval, shape functions for infinite elements are singular at the infinite boundary of the element, and GLL quadrature cannot be used.

To circumvent the singularity, we can use either Gauss-Legendre quadrature, which does not include the end points of the interval, or Gauss-Radau (GR) quadrature, which includes only the nearest end point. We choose to use the GR quadrature so that the furthest end lies at infinity and the nearest end coincides with the spectral element, as shown in Fig. 7. GR quadrature is given by

$\int_{-1}^{1} f(\xi) \mathrm{d} \xi=w_{1} f(-1)+\sum_{\alpha=2}^{n} w_{\alpha} f\left(\xi_{\alpha}\right)$

It is exact for polynomials up to order $2 n-2$. Based on this expression, infinite-element shape functions can be safely computed at all quadrature points. By combining GR quadrature in infinite elements with GLL quadrature in spectral elements, quadrature points on a spectral-infinite-element interface coincide (Fig. 7). This coincidence naturally and elegantly couples spectral and infinite elements.

\section{PARALLELIZATION}

We use non-overlapping domain decomposition for parallelization, in which each partition contains a unique set of elements, and nodes are only shared on interfaces. Since pre- and post-processing of infinite elements is similar to spectral elements, infinite elements do not pose any difficulty for parallelization. Depending on the geometry of the domain decomposition, an infinite element and its pole may lie in different partitions, which also does not pose any problem for parallelization. The Message Passing Interface (MPI) is used as the parallel library (e.g. Gropp et al. 1994). We use the mesh partitioning tool SCOTCH (Pellegrini \& Roman 1996) to partition the mesh. We implemented parallel iterative Krylov solvers using PETSc, a portable and extensible toolkit for scientific computation (Balay et al. 2015). 


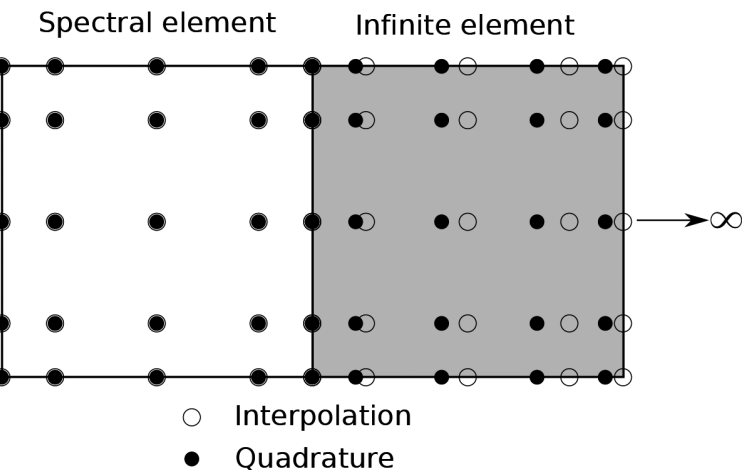

Figure 7. Coupling between a spectral and an infinite element in 2-D. Both elements use identical interpolation nodes. Spectral elements use Gauss-LobattoLegendre (GLL) quadrature points, whereas infinite elements use Gauss-Radau (GR) quadrature points in the infinite direction and GLL quadrature in the two remaining directions. GLL and GR quadrature points coincide on a spectral-infinite-element boundary.

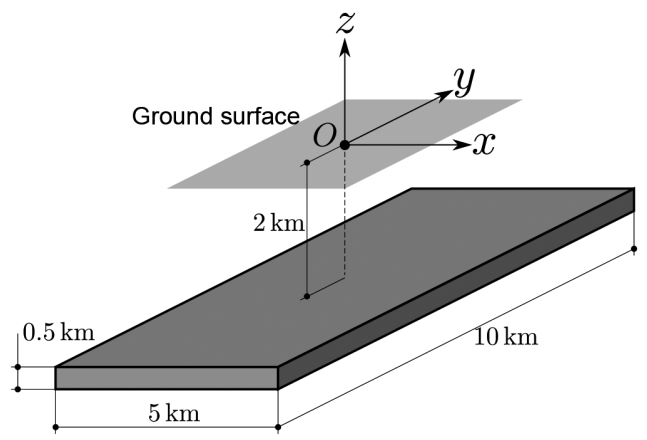

Figure 8. Model geometry for a density anomaly in the shape of a rectangular prism.

\section{NUMERICAL EXAMPLES}

We rely on MeshAssist (Gharti et al. 2017) and Trelis/CUBIT (CUBIT 2017) for model preparation and meshing for all examples included in this article. Each spectral or infinite element involves 3 GLL or GR points in each direction, that is, a total number of $n=27$ points per element. Since all elements of the mesh have planar faces, we use only the 8 corner nodes for the geometrical mapping of an spectral element, that is, the number of geometrical nodes is $n_{g}=8$. For an infinite element, we use effectively $n_{g}=5$ - pole generally taken at the centre of mass and four intermediate corner nodes such that each intermediate corner node is determined by the relation $x_{2}=x_{0}+\gamma\left(x_{1}-x_{0}\right)$, as described in Section 2.3. Of course there are eight nodes, in principle, but four nodes have the same coordinates as the pole. We set $\gamma=2$, and we use a conjugate gradient solver with a geometric algebraic multigrid (AMG) pre-conditioner (e.g. Stüben 2001), with a relative tolerance of $10^{-7}$.

\subsection{Rectangular prism}

For the first example, we consider a rectangular prism of size $5 \mathrm{~km} \times 10 \mathrm{~km} \times 0.5 \mathrm{~km}$ with a density anomaly $\delta \rho=400 \mathrm{~kg} \mathrm{~m}^{-3}$ located at a depth of $2 \mathrm{~km}$ from the ground surface, as shown in Fig. 8. We consider a gravity anomaly profile on the ground surface along the $x$-axis from $-15 \mathrm{~km}$ to $15 \mathrm{~km}$. We created a model of size $30 \mathrm{~km} \times 20 \mathrm{~km} \times 6.5 \mathrm{~km}$ which sufficiently covers the prism and the observation points. We extended the model slightly above the ground surface to avoid placing the infinite-element layer adjacent to observation points.

Initially, we selected a coarse element size of $2 \mathrm{~km}$ and meshed the model using hexahedral elements. The mesh consists of only 825 elements and honors the prism, as shown in Fig. 9(left-hand panel). A single layer of infinite elements is added outside the mesh domain, as shown in Fig. 9(right-hand panel). We position the pole for the decay function required by the infinite elements at the centre of the prism, which is also the centre of mass. Opposite faces of infinite elements must be parallel or diverging with respect to the pole so that they do not converge at infinity.

An analytical expression for the gravity anomaly of the prism is given by eq. (A1). Fig. 10 shows a gravity anomaly profile computed at the ground surface, $z=0$. We observe good agreement with the analytical result. Although we used a very coarse mesh, we obtain acceptable results because the mesh perfectly honors the prism.

Next, we refined the mesh by decreasing the element size from $2 \mathrm{~km}$ to $1 \mathrm{~km}$. The refinement increases the number of elements to 6600. The resulting profile for the gravity anomaly matches very well with the analytical solution, demonstrating rapid convergence in terms of mesh refinement. Finally, we plot the computed gravitational potential and the vertical component of perturbed gravitational acceleration in 3-D, as shown in the Fig. 11. As expected, the potential is maximum at the centre of mass and decays away from the prism. On the other 

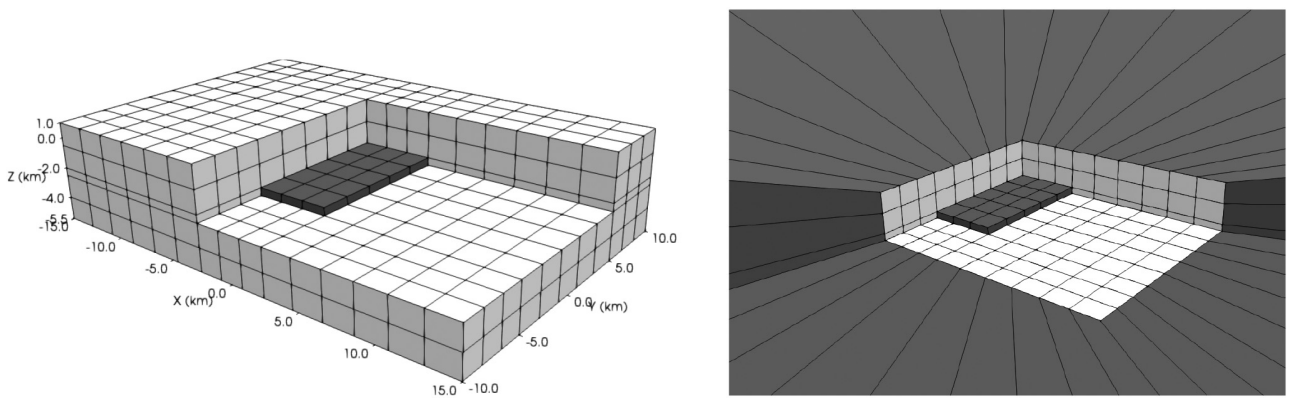

Figure 9. Left-hand panel: spectral-element mesh of a model with a buried rectangular prism. The model is sectioned to visualize the prism (dark grey). Right-hand panel: infinite elements (outer dark grey) radiating from the outer surfaces of the full simulation domain. Only infinite elements from the visible outer surfaces are shown for clarity.

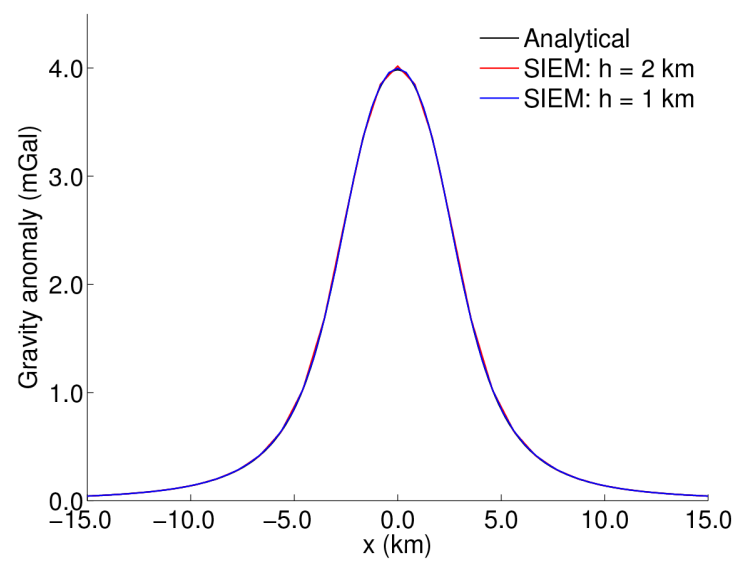

Figure 10. Profile for the vertical component of the gravity anomaly at $z=0$ for a rectangular prism.
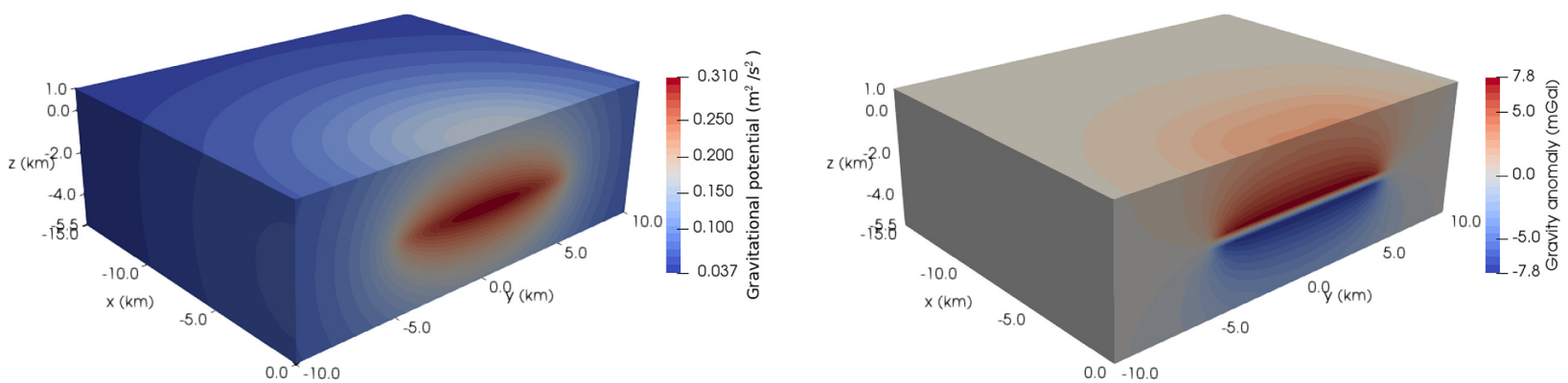

Figure 11. Left-hand panel: gravitational potential for a rectangular prism. Right-hand panel: vertical component of the perturbed gravitational acceleration.

hand, the gravity anomaly is zero at the centre of mass. Its magnitude is maximum on either side of the centre of mass in the prism, and it decays away from the prism. Due to the positive density anomaly, we observe a positive gravity anomaly at the ground surface.

\subsection{Spherical salt dome}

In this section, we consider a spherical salt dome of radius $4 \mathrm{~km}$ buried at a depth of $z=6 \mathrm{~km}$, as shown in Fig. 12 . This example closely resembles the salt dome located $\approx 125$ miles southeast of Galveston, TX, USA, near the outer edge of the continental shelf (Nettleton 1957 ). The salt dome and the host sediment have average densities of 2200 and $2400 \mathrm{~kg} \mathrm{~m}^{-3}$, respectively, resulting in a negative density anomaly of $\delta \rho=-200 \mathrm{~kg} \mathrm{~m}^{-3}$ in the salt dome.

We created a model of size $40 \mathrm{~km} \times 30 \mathrm{~km} \times 30 \mathrm{~km}$ that covers the dome and the observation points. For accuracy, we slightly extended the model above the ground surface to avoid placing the infinite element layer adjacent to observation points. We mesh the model using hexahedral elements of size $1 \mathrm{~km}$ inside the dome and $2 \mathrm{~km}$ outside the dome. The mesh consists of 6702 elements and honors the spherical dome. We add an infinite-element layer outside the model surface based on a pole positioned at the centre of the dome, which is also the centre of mass for the model. The analytical expression for the gravity anomaly due to the sphere is given by eq. (A4).

Fig. 14 shows the gravity anomaly profile calculated at the ground surface, $z=0$, along the $x$-axis from $-20 \mathrm{~km}$ to $20 \mathrm{~km}$. Due to the 


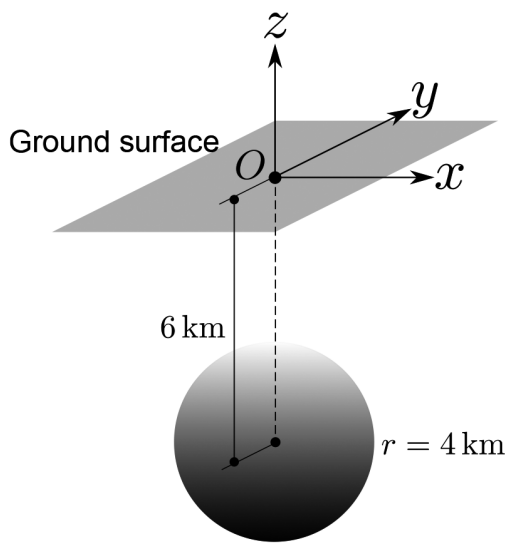

Figure 12. Model geometry for a spherical salt dome.

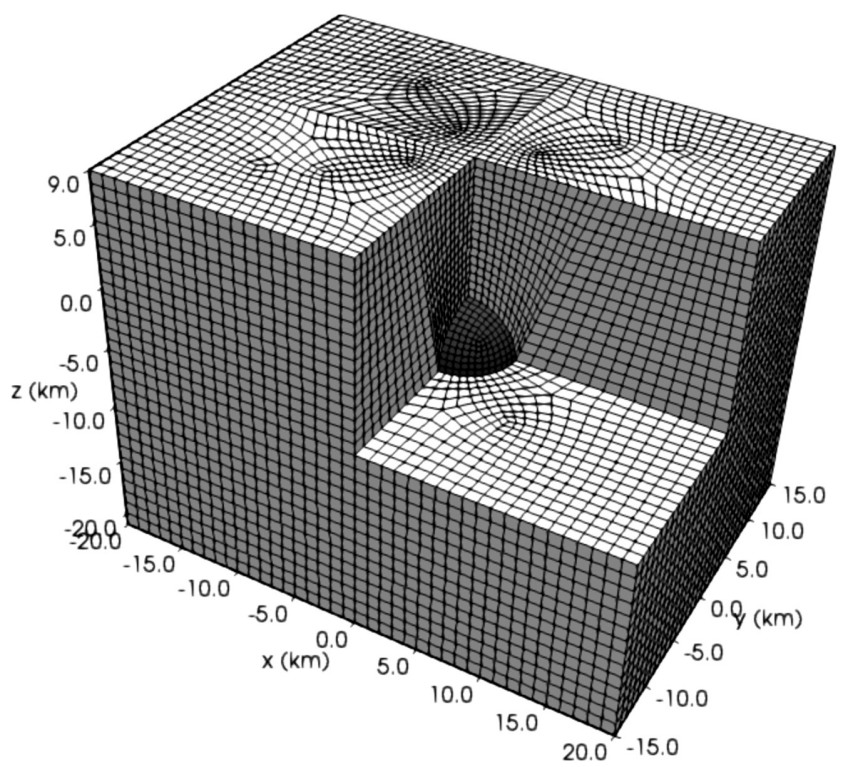

Figure 13. Spectral-element mesh for a spherical salt dome. The model is sectioned to visualize one quadrant of the salt dome. The salt dome mesh is finer than the surrounding rock.

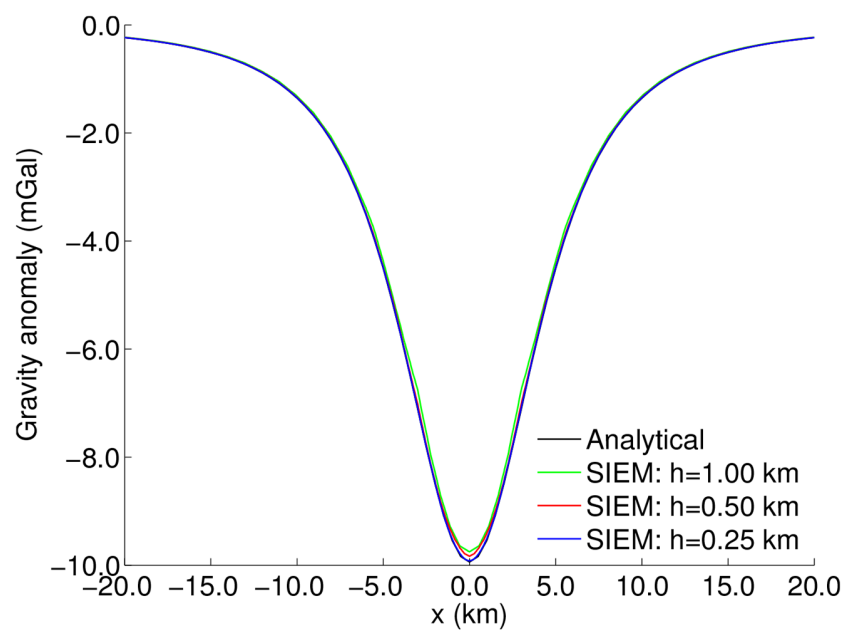

Figure 14. Profile for the vertical component of gravity at $z=0$ along the $x$-axis for a spherical salt dome. 

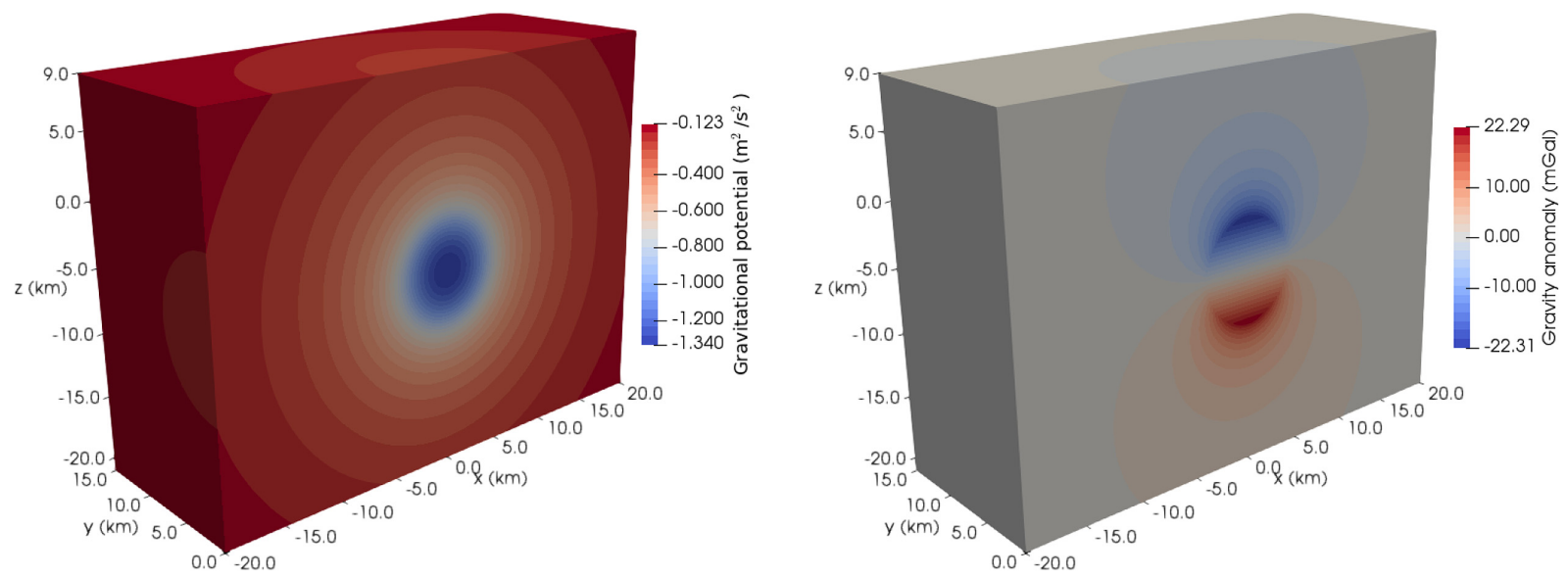

Figure 15. Left-hand panel: gravitational potential for a spherical salt dome. Right-hand panel: vertical component of the perturbed gravitational acceleration.

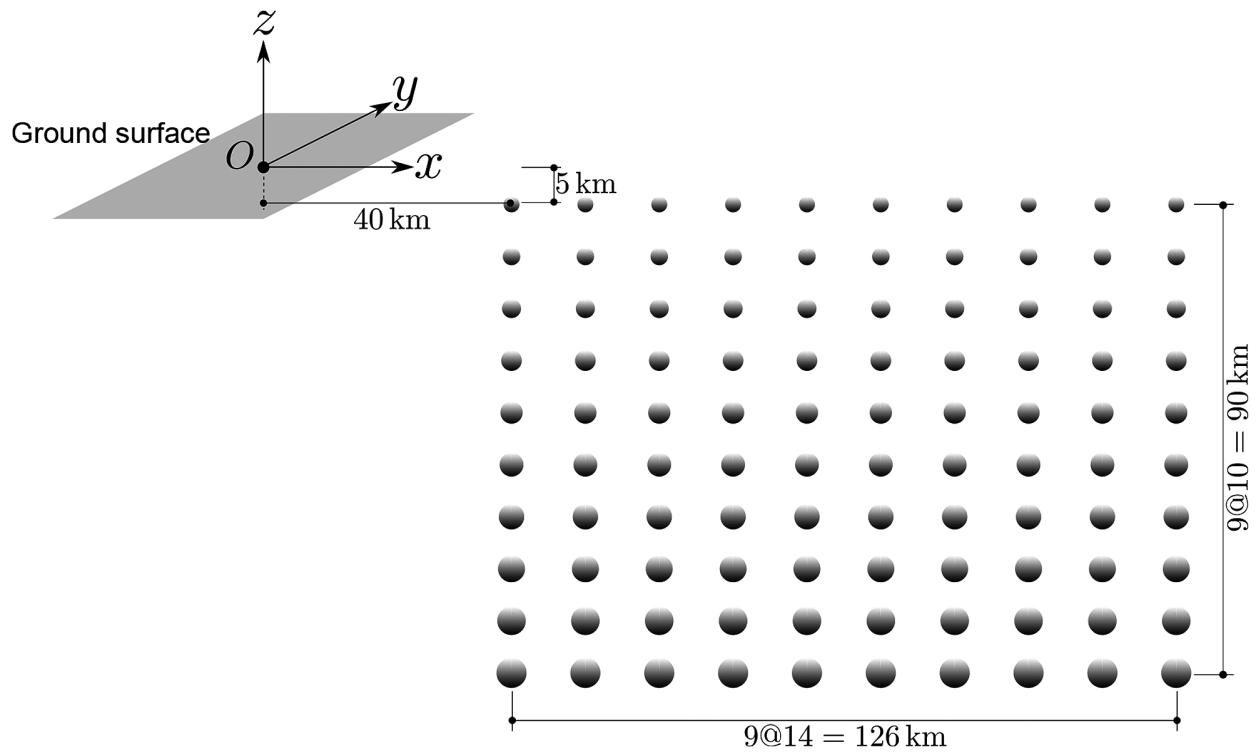

Figure 16. Layout of an array of 100 spheres of varying sizes.

negative density anomaly, we observe a negative gravity anomaly. The computed result is in good agreement with the analytical solution, but not as good as in the case of the prism. The largest discrepancy is around the centre of the dome.

We refined the mesh by decreasing the element size to 0.5 and $1 \mathrm{~km}$, respectively, inside and outside the dome, which increases the number of elements to 53615 . The resulting gravity anomaly profile is closer to the analytical result, but we still observe some discrepancies near the centre. We further refined the mesh with element sizes of 0.25 and $0.5 \mathrm{~km}$, respectively, inside and outside the dome, thereby increasing the total number of elements to 156538 . This refinement gives a nearly perfect match with the analytical solution. The spherical geometry poses a challenge for the mesher, because the coarser meshes fail to properly capture the anomaly. Compared to the prism discussed in the previous section, a finer mesh is needed to accurately represent a spherical anomaly.

In Fig. 15, we plot the gravitational potential and the vertical component of the perturbed gravitational acceleration in 3-D. The potential is minimal but largest in magnitude at the dome centre, where the gravity anomaly is zero. The magnitude of the gravity anomaly is largest on the either sides of the centre of mass. Both the potential and the gravity anomaly decay away from the dome at the expected rate.

\subsection{Multiple spheres with varying size and density}

In this section, we design a problem similar to the example in Martin et al. (2017). We consider 100 spheres with varying radii and density anomalies in a vertical plane, as shown in Fig. 16. The model consists of 10 horizontal equidistant lines in the vertical plane with a vertical spacing of $10 \mathrm{~km}$. Each horizontal line consists of 10 equidistant spheres with a horizontal spacing of $14 \mathrm{~km}$. The $l$ th horizontal line has a constant sphere radius, $r_{l}$, and density, $\rho_{l}$, determined by the relations, $r_{l}=2+0.2(l-1) \mathrm{km}$, and $\rho_{l}=50(1+4 l) \mathrm{kg} \mathrm{m}^{-3}$, respectively, such that lines $l=1$ to 10 are numbered top to bottom. The first sphere of the first horizontal line is located at $x=40 \mathrm{~km}, y=0 \mathrm{~km}$ and $z=$ $-5 \mathrm{~km}$ from the origin. 

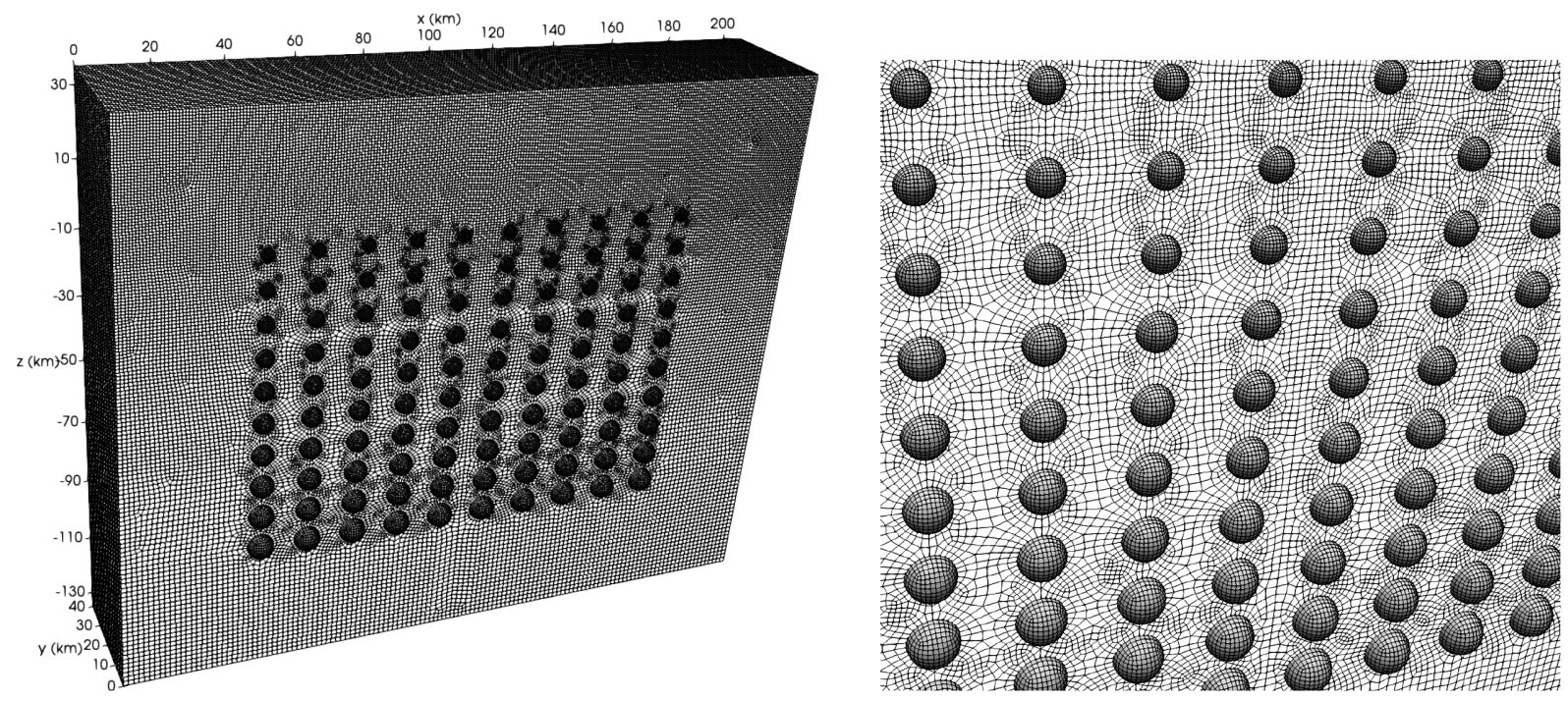

Figure 17. Left-hand panel: spectral-element mesh for an ensemble of 100 spheres. Right-hand panel: zoom in view of the mesh.

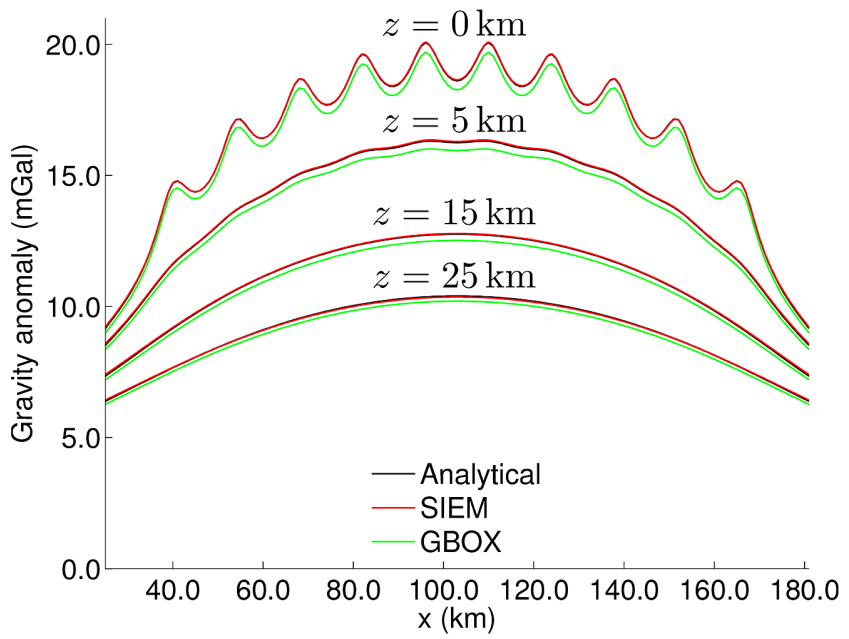

Figure 18. Profiles of the vertical component of the gravity anomaly at various elevations for an ensemble of spheres.

We created a model of size $206 \mathrm{~km} \times 80 \mathrm{~km} \times 170 \mathrm{~km}$ covering all spheres and observation points. The outer surfaces of the model lie $40 \mathrm{~km}$ in each direction from the nearest sphere centre. We selected an element size of $0.2 r_{s}$ inside a sphere and $0.4 r_{s}$ outside a sphere, where $r_{s}$ is the sphere's radius. This approach gradually coarsens the mesh from top to bottom, varying the element size inside the sphere from 0.4 to $0.76 \mathrm{~km}$ and outside the sphere from 0.8 to $1.52 \mathrm{~km}$. This approach ensures the same number of elements in all spheres (Fig. 17). Although not strictly necessary, this maintains a relatively uniform resolution for all spheres. We positioned the pole of the infinite elements at the centre of mass of the model. The mesh consists of 2886000 spectral elements, for a total of 22808007 degrees of freedom. We used 256 processors, on which it took about $8 \mathrm{~min}$ to compute the gravity anomaly.

As a benchmark, we also computed the gravity anomalies using GBOX (Blakely 1995). We discretized the model using uniform cubic cells. Since the smallest spectral element has a size of $0.4 \mathrm{~km}$ and each spectral element has three GLL points in each direction, we chose a cell size of $0.2 \mathrm{~km}$. Consequently, the GBOX grid resolution is similar to the finest region of the spectral-element mesh, and hence the GBOX model is sampled more finely than the spectral-element model. There are a total of 1611488 cells with non-zero density anomaly. We set 157 observation points each at four elevations, namely, $0,5,15$ and $25 \mathrm{~km}$, along the $x$-axis, resulting in a total of 628 observation points. The calculations took about $28 \mathrm{~min}$ on a single processor. Since the computational cost for the GBOX solution is proportional to the number of observation points for a given discretization, the total computational cost for the 22808007 points, that is, the total number of points in the spectral-element mesh, would be huge. On the other hand, the total computational cost for the SIEM is independent of the number of observation points for a given discretization.

We plot gravity anomaly profiles at several elevations along the $x$-axis, specifically at $0,5,15$ and $25 \mathrm{~km}$, as shown in Fig. 18 . For all profiles, we observe excellent agreement of the SIEM solution with the analytical solution given by eq. (A5). At zero elevation, we clearly observe the influence of individual spheres. As we move away from the spheres, individual influences diminish until all spheres behave as a single entity. Although the GBOX model was sampled more finely than the spectral-element model, the resulting gravity anomaly profiles 

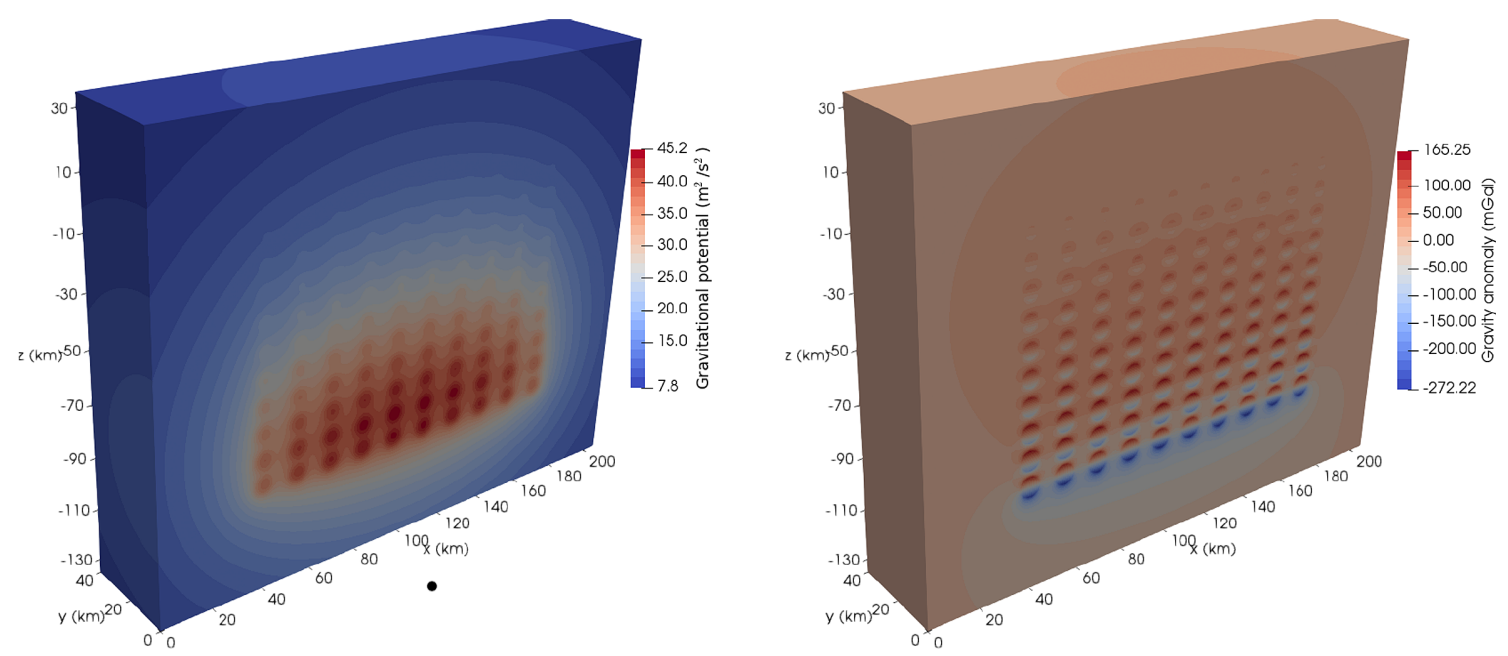

Figure 19. Left-hand panel: gravitational potential for an ensemble of spheres. Right-hand panel: vertical component of the gravity anomaly.

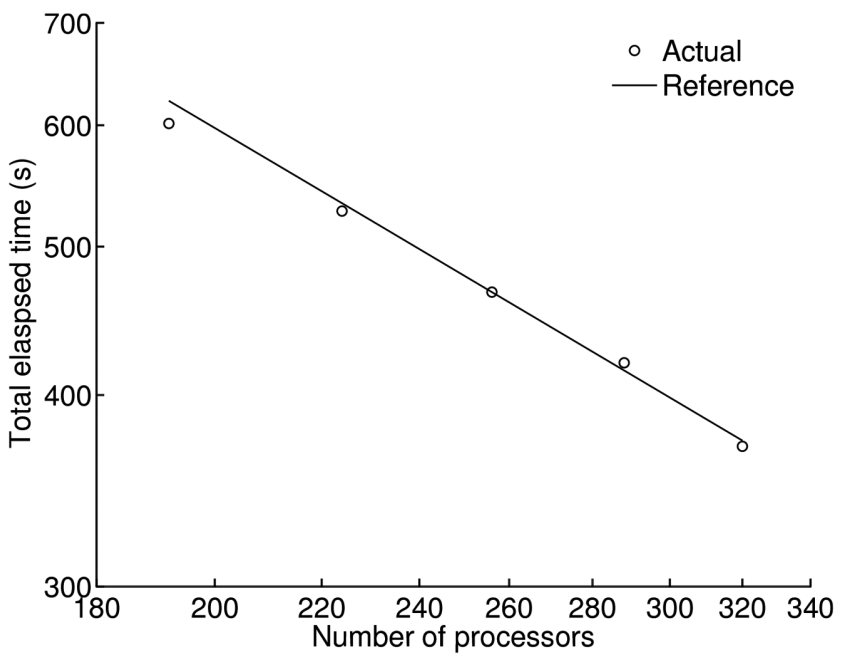

Figure 20. Strong scaling test: total elapsed time for a fixed problem size run on 192, 224, 256, 288 and 320 processors, compared with a reference line computed using the total elapsed time for 256 processors, assuming perfect linear scaling.

are significantly different from the analytical and SIEM results, as shown in Fig. 18. The discrepancies in the GBOX results are due to the variable density anomalies, and the fact that uniform cubic cells are unable to represent spherical shapes accurately.

In Fig. 19, we plot the gravitational potential and gravity anomaly in 3-D. We clearly observe the imprint of individual spheres. The larger spheres and spheres nearer to the centre of mass have stronger potentials, as revealed by the local maxima. Overall, the potential has its largest amplitude around the centre of mass of the entire model. We see positive and negative gravity anomalies on either side of each sphere. The magnitude of the gravity anomaly is largest around the centre of mass of the model. Both the gravity anomaly and the potential decay away from the spheres.

Finally, we perform a simple strong scaling test for the parallel performance of our software package running the same example with different numbers of processors, specifically, 192, 224, 256, 288 and 320 CPUs. Fig. 20 shows the total elapsed time for each run, illustrating that the software package scales very well when compared to the ideal reference.

\subsection{Rectangular prism with a parabolic density distribution}

In this example, we consider a prism of size $2 \mathrm{~km} \times 1 \mathrm{~km} \times 1 \mathrm{~km}$ located at a depth of $1 \mathrm{~km}$ below the ground surface, as shown in Fig. 21. The prism has a parabolic density anomaly distribution as defined in Chakravarthi et al. (2002), namely,

$\delta \rho(z)=\frac{\delta \rho_{0}^{3}}{\left(\delta \rho_{0}-\alpha z\right)^{2}}$.

Here $\delta \rho_{0}$ denotes the density anomaly observed at the ground surface, and $\alpha$ a constant expressed in units of density gradient, $\mathrm{kg} \mathrm{m}^{-3} \mathrm{~m}^{-1}$. We set $\alpha=0.001$. In eq. (31) $z$ is positive below the ground surface, to match the convention used for the analytical solution given by eq. (A6). 


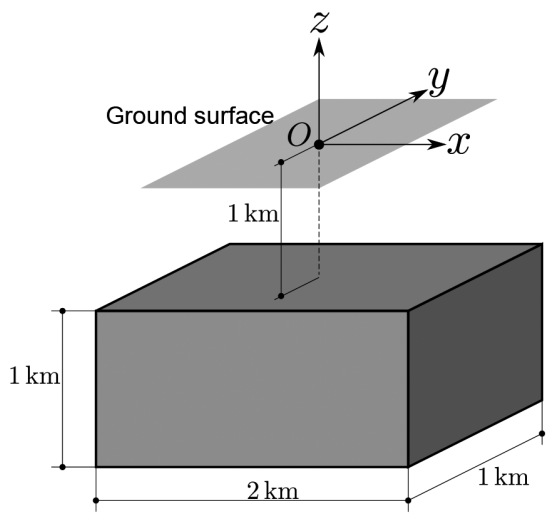

Figure 21. Model geometry involving a buried rectangular prism with a parabolic density anomaly distribution.

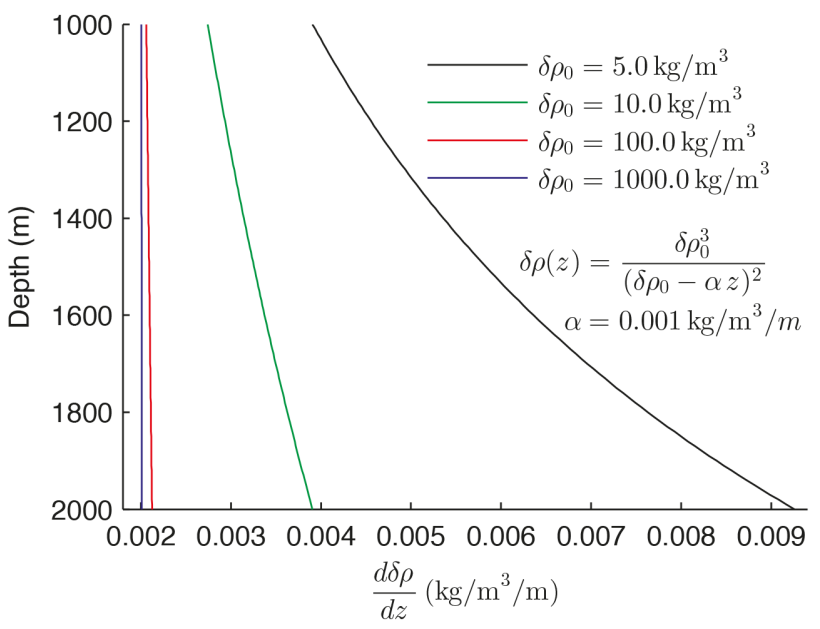

Figure 22. Parabolic density anomaly gradients inside a buried rectangular prism.

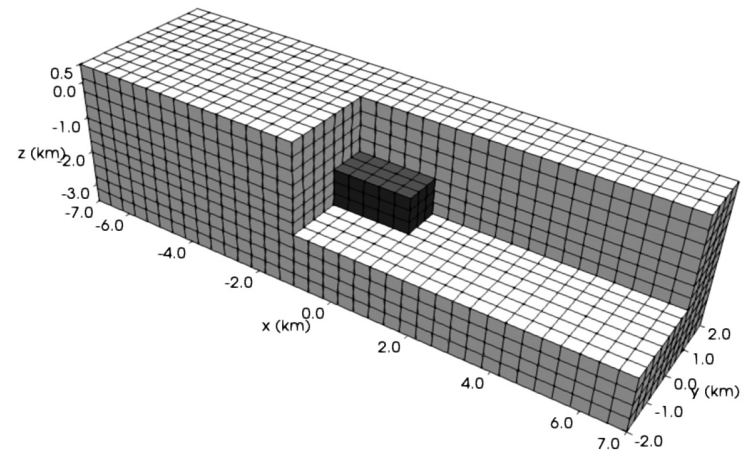

Figure 23. Spectral-element mesh for a model with a rectangular prism with a parabolic density distribution. The model is sectioned to visualize the prism (dark grey).

We used a model of size $14 \mathrm{~km} \times 4 \mathrm{~km} \times 4 \mathrm{~km}$, which covers the prism and observation points. The model is extended above the ground surface by $0.5 \mathrm{~km}$. To begin with, we selected an average element size of $0.4 \mathrm{~km}$. The mesh consists of 4235 spectral elements and honors the prism, as shown in Fig. 23. We consider four different density anomalies, namely, $\delta \rho_{0}=5,10,100$ and $1000 \mathrm{~kg} \mathrm{~m}^{-3}$. Lower values of $\delta \rho_{0}$ give a strongly variable density contrasts, as shown in Fig. 22.

We intentionally position the pole at the centre of the model, and we added the infinite element layer to the external surfaces with respect to the chosen pole position. We note that the centre of mass shifts for the various density profiles, and the pole shifts accordingly. Fig. 24 shows surface gravity anomaly profiles along the $x$-axis from -7 to $7 \mathrm{~km}$ for all cases. Despite the coarse mesh, we observe very good agreement with the analytical result. There is only a small discrepancy around the centre. Next, we set the average element size to $0.2 \mathrm{~km}$ and remesh, thereby increasing the number of spectral elements to 33880 . The resulting gravity anomaly profiles are in excellent agreement with the corresponding analytical results for all density anomaly contrasts. This demonstrates that the spectral-infinite-element method is highly 

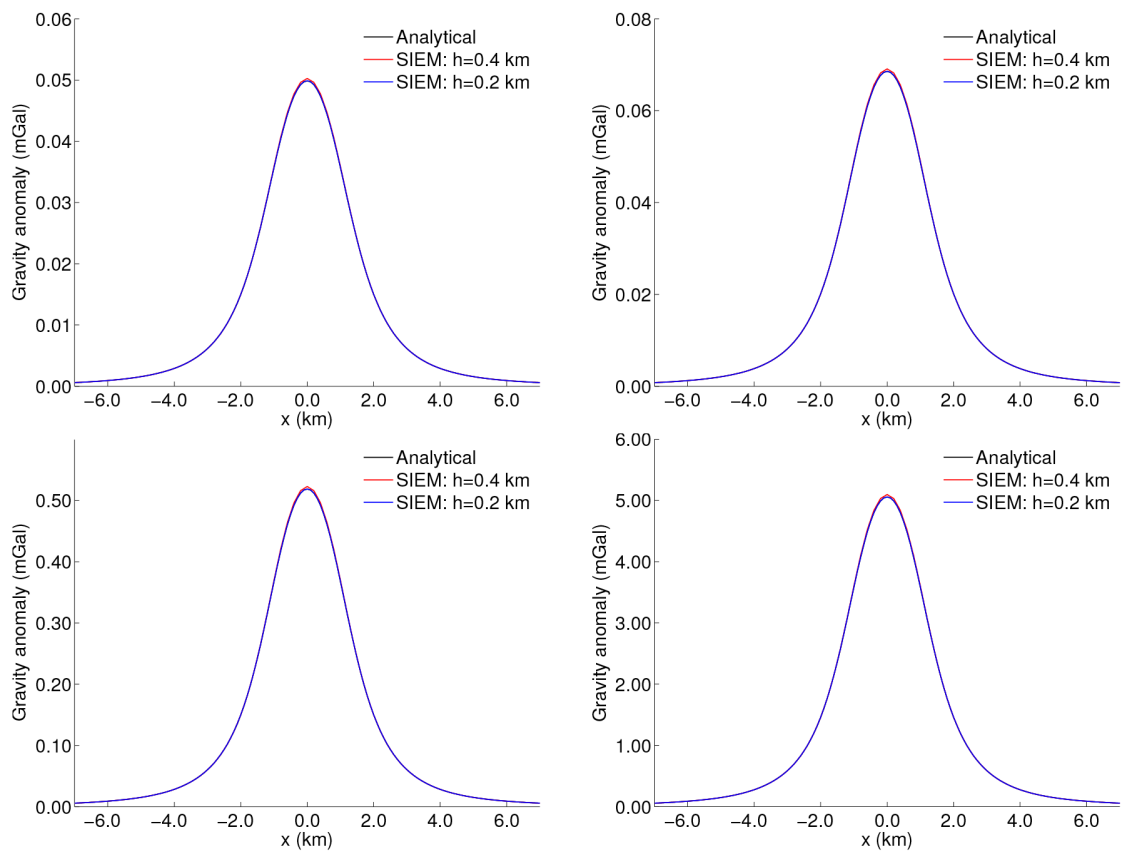

Figure 24. Profiles for the vertical component of the gravity anomaly at $z=0$. Top left-hand panel: $\delta \rho=5 \mathrm{~kg} \mathrm{~m}^{-3}$. Top right-hand panel: $\delta \rho=10 \mathrm{~kg} \mathrm{~m}{ }^{-3}$. Bottom left-hand panel: $\delta \rho=100 \mathrm{~kg} \mathrm{~m}^{-3}$. Bottom right-hand panel: $\delta \rho=1000 \mathrm{~kg} \mathrm{~m}^{-3}$.
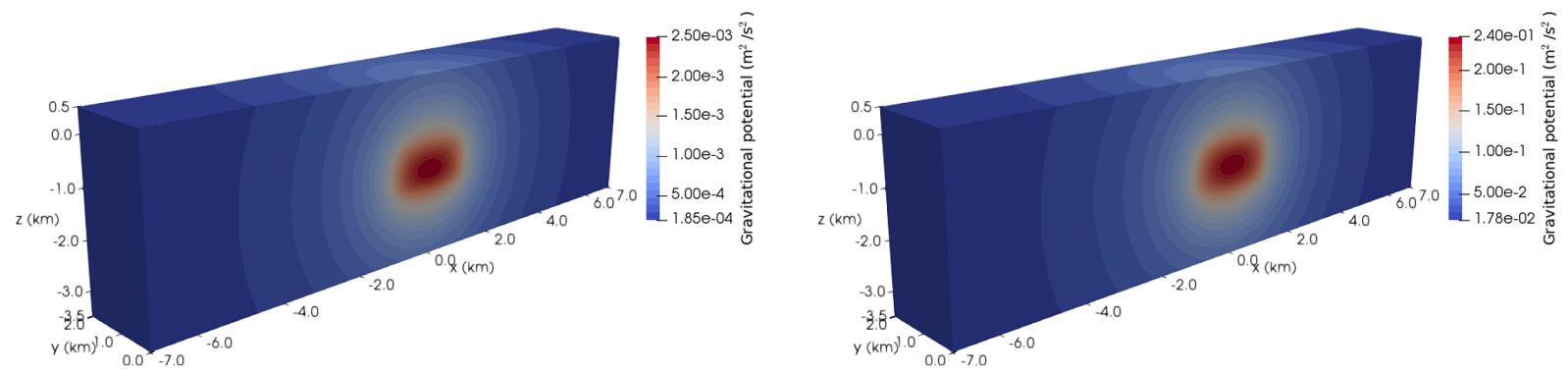

Figure 25. Gravitational potential for a rectangular prism with a parabolic density distribution. Left-hand panel: $\delta \rho=5$ kg m ${ }^{-3}$. Right-hand panel: $\delta \rho=$ $1000 \mathrm{~kg} \mathrm{~m}^{-3}$.
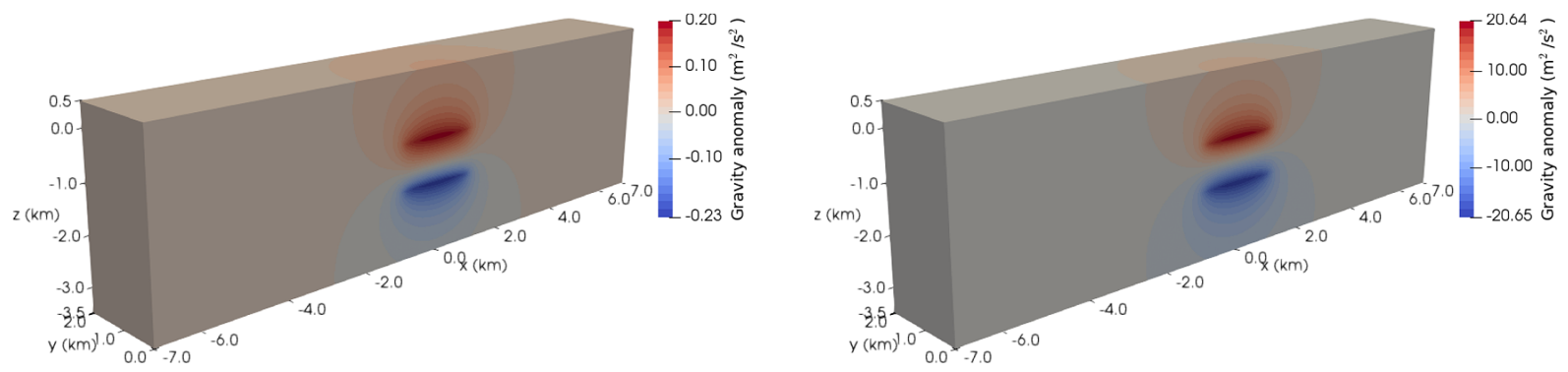

Figure 26. Vertical component of the gravity anomaly for a rectangular prism with a parabolic density distribution. Left-hand panel: $\delta \rho=5 \mathrm{~kg} \mathrm{~m}{ }^{-3}$. Right-hand panel: $\delta \rho=1000 \mathrm{~kg} \mathrm{~m}^{-3}$.

accurate even for strongly variable density contrasts. Furthermore, the results demonstrate that slightly shifting the pole position does not affect accuracy.

Figs 25 and 26 show 3-D plots of the gravitational potential and gravity anomaly, respectively. Since we observe similar patterns for all density contrasts, we plot only the smallest and largest density contrasts, namely, $\delta \rho=5 \mathrm{~kg} \mathrm{~m}^{-3}$ and $\delta \rho=1000 \mathrm{~kg} \mathrm{~m}^{-3}$. They are similar in pattern but differ in magnitude. The potential is maximal around the mass centre and decays away from the prism, whereas the gravity anomaly is zero around the centre of mass, maximal around the prism edges, and also decays away from the prism. 

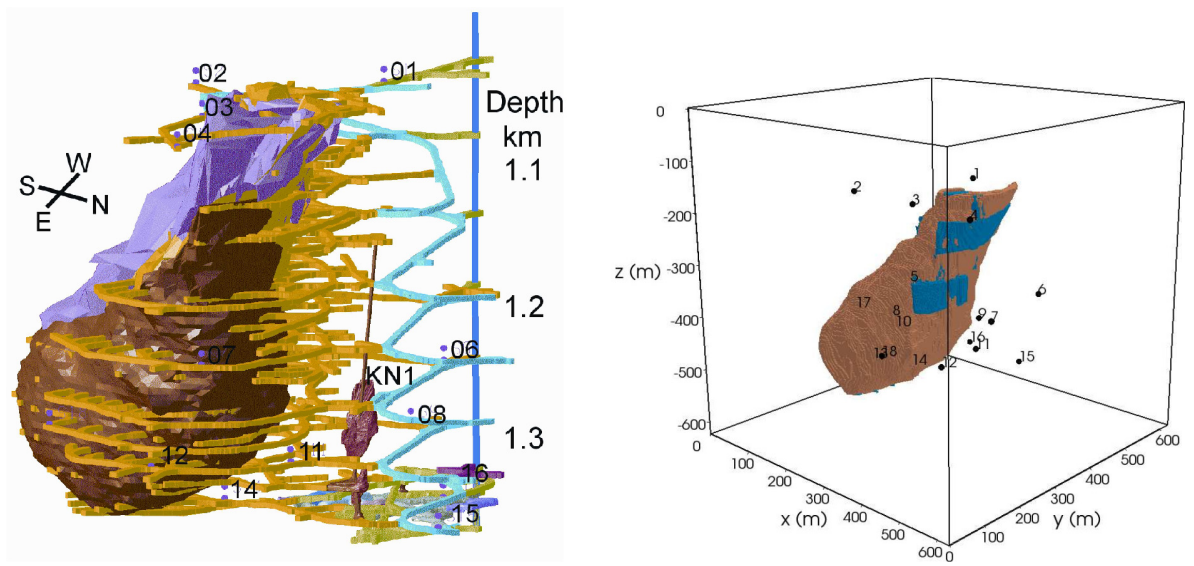

Figure 27. Left-hand panel: Pyhäsalmi mine with surrounding infrastructure: copper/zinc ore body (brown/pink), access tunnels (yellow), elevator shaft (dark blue), and seismic stations (numbered). The passage for quarried ore is marked by KN1. Right-hand panel: 3-D density model (see Table 1) of the Pyhäsalmi mine: stopes (i.e. mined out voids, blue) and ore body (brown). The remainder is host rock. Geophones are indicated by black dots and numbered.
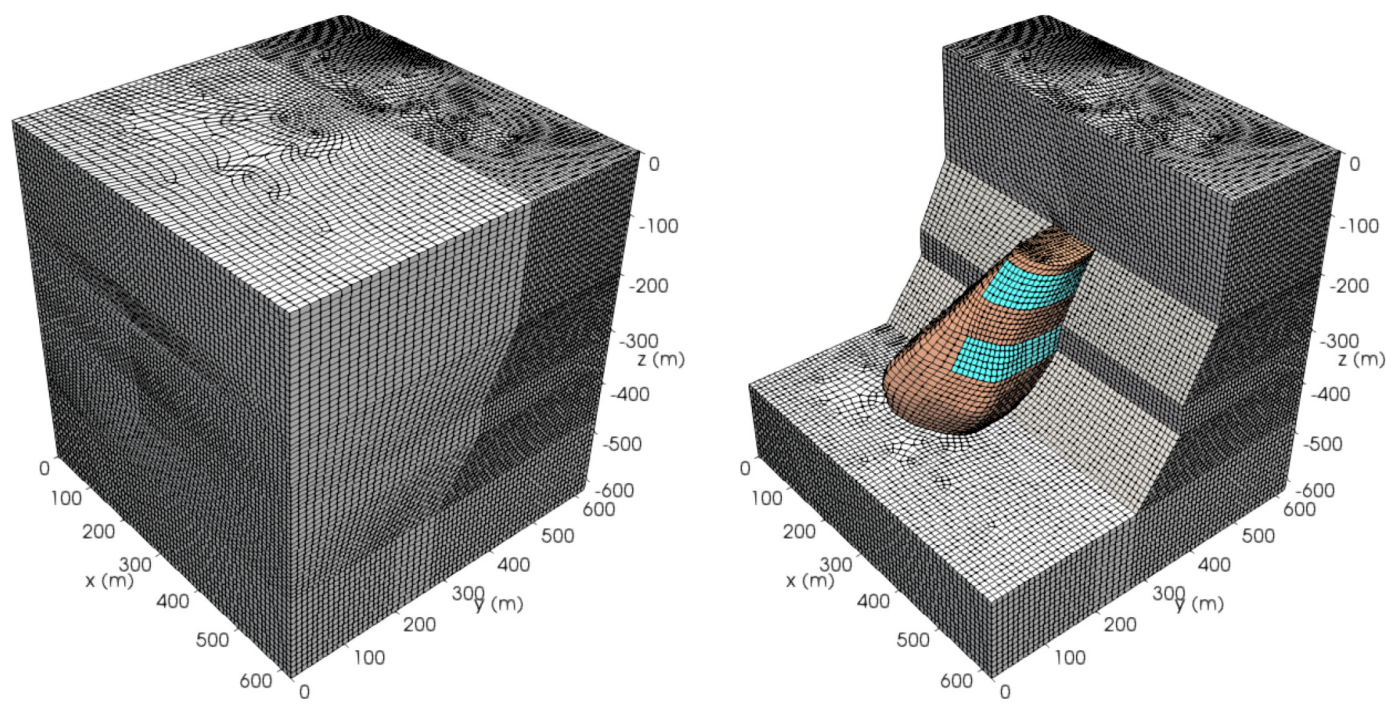

Figure 28. Left-hand panel: spectral-element mesh for the Pyhäsalmi mine model. Right-hand panel: interior section of the mesh visualizing the ore body (brown) and stopes (cyan).

\subsection{Application to an underground ore mine}

To demonstrate our method for realistic problems, we compute the gravity anomaly of an existing underground ore mine, namely, the Pyhäsalmi mine in Finland. This active, deep mine contains volcanogenic massive sulphide (VMS) deposits with copper and zinc ore bodies (Puustjärvi 1999). The complete mine layout and surrounding infrastructure are shown in Fig. 27(left-hand panel). For more details on the mine and its microseismic event characteristics, see Oye et al. (2005). Fig. 27(right-hand panel) shows the $622 \mathrm{~m} \times 622 \mathrm{~m} \times 622 \mathrm{~m} 3$-D density model, which consists of host rock, ore body and stopes (i.e. mined out voids). Due to the mine's complex geometry and high density contrasts, it is a very challenging problem to calculate the gravity anomaly.

We meshed the model using an average element size of $10 \mathrm{~m}$. Due to the complex geometry, actual element sizes may differ in some areas, as shown in Fig. 28. The mesh involves 294500 spectral elements and 2517769 degrees of freedom. We used 96 processors for the parallel simulation, which took $35 \mathrm{~s}$.

Fig. 29(left-hand panel) shows the gravitational potential in a 3-D section. It is maximal around the centre of mass and decays away from the ore body. Similarly, Fig. 29(right-hand panel) shows the gravity anomaly in the same 3-D section. We observe negative anomalies around voids and below the approximate centre of mass. The gravity anomaly observed on the top surface is positive due to the larger positive density anomaly caused by the ore body. 

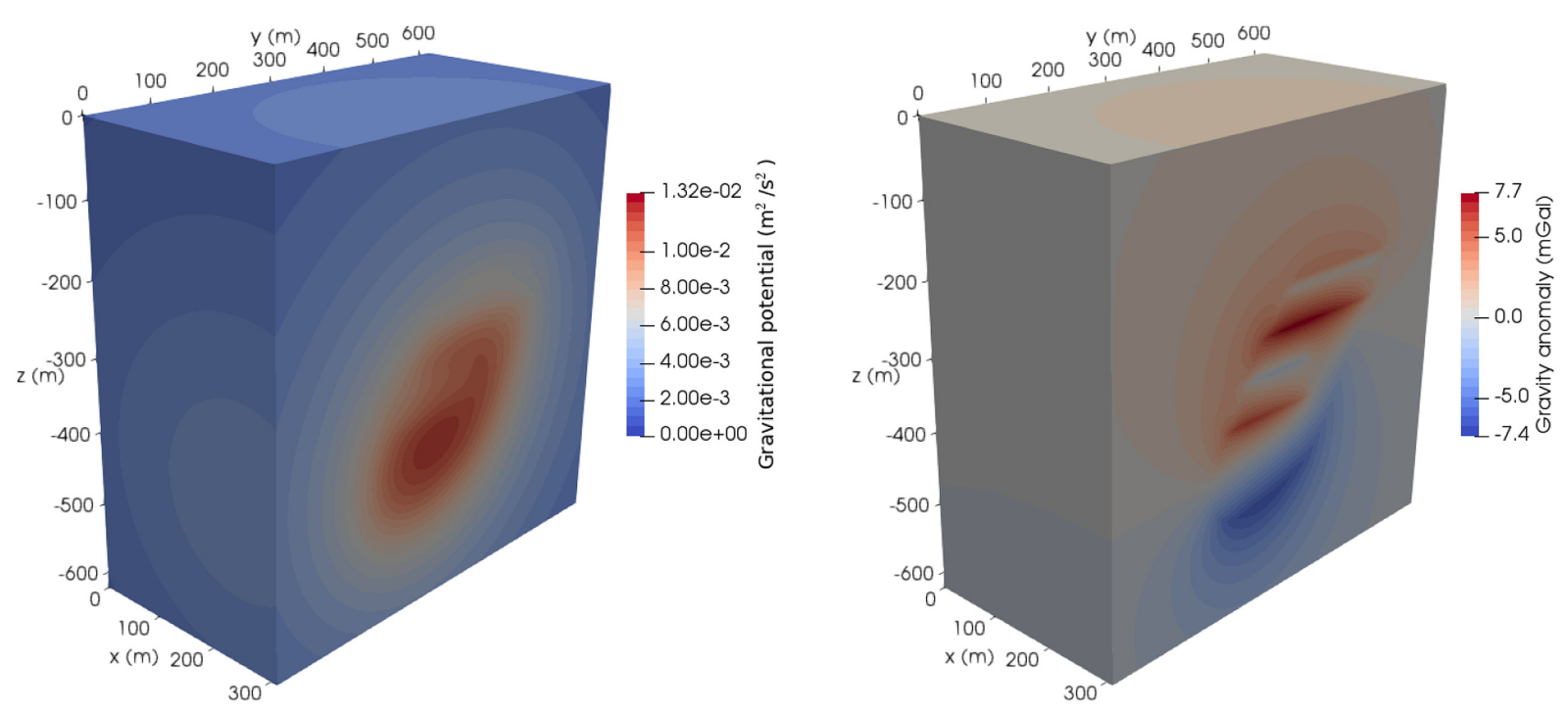

Figure 29. Left-hand panel: gravity potential for the Pyhäsalmi mine. Right-hand panel: vertical component of the gravity anomaly.

\section{CONCLUSIONS}

We have successfully implemented a spectral-infinite-element method to solve the unbounded Poisson/Laplace equation for gravity anomalies induced by density heterogeneities. We benchmarked our results with analytical solutions for a range of problems, and used the Pyhäsalmi mine as a concrete example.

Our tests shows that the method is highly accurate and efficient, even in the presence of strongly variable density anomalies. The additional computational cost and memory requirement associated with a single layer of infinite elements is insignificant. Since the computational cost is independent of the number of observation points, the SIEM is well suited for very large models and a large number of observation points. A simple strong-scaling performance test shows that the software is scalable and efficient; nevertheless, GPU acceleration will be of future interest.

The main advantage of this numerical discretization of the unbounded Poisson equation is that it can be naturally coupled with the the laws of continuum mechanics that govern geostatic and geodynamic deformations. This allows us to solve the coupled elastic-gravitational equations efficiently to address several challenging geophysical problems, such as long-period seismic wave propagation, glacial-isostatic adjustment, post-seismic relaxation and tidal loading. Future work will focus on these important problems.

\section{ACKNOWLEDGEMENTS}

We thank Volker Oye, Katja Sahala, and ISS for access to the mine model. We thank Frederik J. Simons for helpful discussions. Parallel programs were run on computers provided by the Princeton Institute for Computational Science and Engineering (PICSciE). 3D data were visualized using the open-source parallel visualization software ParaView/VTK (www.paraview.org). This research was partially supported by NSF grants 1644826 and 1550901 . Our software is open source and freely available via the Computational Infrastructure for Geodynamics (CIG; geodynamics.org). We thank the editor Prof. Bert Vermeersen, Bernhard Steinberger, David Al-Attar, and an anonymous reviewer for their insightful comments which helped to improve the manuscript.

\section{REFERENCES}

Abdel-Fattah, T.T., Hodhod, H.A. \& Akl, A.Y., 2000. A novel formulation of infinite elements for static analysis, Comput. Struct., 77(4), 371-379.

Al-Attar, D. \& Woodhouse, J., 2008. Calculation of seismic displacement fields in self-gravitating earth models-applications of minors vectors and symplectic structure, Geophys. J. Int., 175(3), 1176-1208.

Angelov, T.A., 1991. Infinite elements-theory and applications, Comput. Struct., 41(5), 959-962.

Balay, S. et al., 2015. PETSc users manual, Tech. Rep. ANL-95/11 - Revision 3.6, Argonne National Laboratory.

Banerjee, B. \& Das Gupta, S.P., 1977. Gravitational attraction of a rectangular parallelepiped, Geophysics, 42(5), 1053-1055.

Beer, G. \& Meek, J.L., 1981. 'Infinite domain' elements, Int. J. Numer. Methods Eng., 17(1), 43-52.

Bettess, P., 1977. Infinite elements, Int. J. Numer. Methods Eng., 11(1), 53-64.
Blakely, R.J., 1995. Potential Theory in Gravity and Magnetic Applications, Cambridge University Press.

Bott, M.H.P., 1960. The use of rapid digital computing methods for direct gravity interpretation of sedimentary basins, Geophys. J. R. astr. Soc., 3(1), 63-67.

Cai, Y. \& Wang, C.-Y., 2005. Fast finite-element calculation of gravity anomaly in complex geological regions, Geophys. J. Int., 162(3), 696708.

Canuto, C., Hussaini, M.Y., Quarteroni, A. \& Zang, T.A., 1988. Spectral Methods in Fluid Dynamics, Springer.

Chakravarthi, V., Raghuram, H.M. \& Singh, S., 2002. 3-D forward gravity modeling of basement interfaces above which the density contrast varies continuously with depth, Comput. Geosci., 28(1), 53-57.

Chaljub, E. \& Valette, B., 2004. Spectral element modelling of threedimensional wave propagation in a self-gravitating Earth with an arbitrarily stratified outer core, Geophys. J. Int., 158(1), 131-141. 
CUBIT, 2017. CUBIT 15.3 User Documentation, Sandia National Laboratories, [Online; accessed 27-Jan-2017].

Curnier, A., 1983. A static infinite element, Int. J. Numer. Methods Eng., 19(10), 1479-1488.

Dahlen, F.A., 1974. On the static deformation of an earth model with a fluid core, Geophys. J. Int., 36(2), 461-485.

Dahlen, F.A. \& Tromp, J., 1998. Theoretical Global Seismology, Princeton University Press.

Deville, M.O., Fischer, P.F. \& Mund, E.H., 2002. High-Order Methods for Incompressible Fluid Flow, Cambridge University Press.

El-Esnawy, N.A., Ak1, A.Y. \& Bazaraa, A.S., 1995. A new parametric infinite domain element, Finite Element. Anal. Des., 19(1-2), 103-114.

Faccioli, E., Maggio, F., Paolucci, R. \& Quarteroni, A., 1997. 2D and 3D elastic wave propagation by a pseudo-spectral domain decomposition method, J. Seismol., 1, 237-251.

García-Abdeslem, J., 2005. The gravitational attraction of a right rectangular prism with density varying with depth following a cubic polynomial, Geophyics, 70(6), J39-J42.

Gharti, H.N. \& Tromp, J., 2017. A spectral-infinite-element solution of Poisson's equation: an application to self gravity, preprint (arXiv: 1706.00855)

Gharti, H.N., Komatitsch, D., Oye, V., Martin, R. \& Tromp, J., 2012a. Application of an elastoplastic spectral-element method to 3D slope stability analysis, Int. J. Numer. Methods Eng., 91, 1-26.

Gharti, H.N., Oye, V., Komatitsch, D. \& Tromp, J., 2012b. Simulation of multistage excavation based on a 3D spectral-element method, Comput. Struct., 100-101, 54-69.

Gharti, H.N., Langer, L., Roth, M., Tromp, J., Vaaland, U. \& Yan, Z., 2017. MeshAssist: An Open-Source and Cross-Platform Meshing Assistant Tool, Zenodo.

Gropp, W., Lusk, E. \& Skjellum, A., 1994. Using MPI, Portable Parallel Programming with the Message-Passing Interface, MIT Press.

Hejlesen, M.M., Rasmussen, J.T., Chatelain, P. \& Walther, J.H., 2013. A high order solver for the unbounded poisson equation, J. Computat. Phys., 252(0), 458-467.

Johnson, L.R. \& Litehiser, J.J., 1972. A method for computing the gravitational attraction of three-dimensional bodies in a spherical or ellipsoidal earth, J. geophys. Res., 77(35), 6999-7009.

Kumar, P., 1985. Static infinite element formulation, J. Struct. Eng., 111(11), 2355-2372.

Marques, J. M. M.C. \& Owen, D. R.J., 1984. Infinite elements in quasi-static materially nonlinear problems, Comput. Struct., 18(4), 739-751.

Martin, R. et al., 2017. A high-order 3-D spectral-element method for the forward modelling and inversion of gravimetric data - application to the western Pyrenees, Geophys. J. Int., 209(1), 406-424.

Mavriplis, C., 1989. Laguerre polynomials for infinite-domain spectral elements, J. Computat. Phys., 80(2), 480-488.

Medina, F. \& Taylor, R.L., 1983. Finite element techniques for problems of unbounded domains, Int. J. Numer. Methods Eng., 19(8), 1209-1226.

Nagy, D., 1966. The gravitational attraction of a right rectangular prism, Geophysics, 31(2), 362-371.
Nettleton, L.L., 1957. Gravity survey over a gulf coast continental shelf mound, Geophysics, 22(3), 630-642.

Oye, V., Bungum, H. \& Roth, M., 2005. Source parameters and scaling relations for mining related seismicity within the Pyhaesalmi ore mine, Finland, Bull. seism. Soc. Am., 95, 1011-1026.

Patera, A.T., 1984. A spectral element method for fluid dynamics: laminar flow in a channel expansion, J. Computat. Phys., 54, 468-488.

Pellegrini, F. \& Roman, J., 1996. SCOTCH: A software package for static mapping by dual recursive bipartitioning of process and architecture graphs, Lecture Notes Comput. Sci., 1067, 493-498.

Peter, D. et al., 2011. Forward and adjoint simulations of seismic wave propagation on fully unstructured hexahedral meshes, Geophys. J. Int., 186(2), $721-739$

Plouff, D., 1976. Gravity and magnetic fields of polygonal prisms and application to magnetic terrain corrections, Geophysics, 41(4), 727-741.

Pollitz, F.F., 1997. Gravitational viscoelastic postseismic relaxation on a layered spherical Earth, J. geophys. Res., 102(B8), 17 921-17941.

Puustjärvi, H., 1999. Pyhaesalmi modeling project, section B. Geology, Tech. rep., Geological Survey of Finland, and Outokumpu Mining Oy.

Seriani, G. \& Oliveira, S.P., 2008. Dispersion analysis of spectral-element methods for elastic wave propagation, Wave Motion, 45, 729-744.

Spada, G. et al., 2004. Modeling earth's post-glacial rebound, EOS, Trans. Am. Geophys. Un., 85(6), 62-64.

Stüben, K., 2001. A review of algebraic multigrid, J. Computat. Appl. Math., 128(1), 281-309.

Talwani, M. \& Ewing, M., 1960. Rapid computation of gravitational attraction of three-dimensional bodies of arbitrary shape, Geophysics, 25(1), 203-225.

Talwani, M., Worzel, J.L. \& Landisman, M., 1959. Rapid gravity computations for two-dimensional bodies with application to the Mendocino submarine fracture zone, J. geophys. Res., 64(1), 49-59.

Tromp, J. \& Mitrovica, J.X., 1999. Surface loading of a viscoelastic Earth-I. General theory, Geophys. J. Int., 137(3), 847-855.

Tromp, J., Komatitsch, D. \& Liu, Q., 2008. Spectral-element and adjoint methods in seismology, Commun. Computat. Phys., 3(1), 1-32.

Tsynkov, S.V., 1998. Numerical solution of problems on unbounded domains. a review, Appl. Numer. Math., 27(4), 465-532.

Turcotte, D.L. \& Schubert, G., 2014. Geodynamics, 3rd edn, Cambridge University Press.

Werner, R.A., 1994. The gravitational potential of a homogeneous polyhedron or don't cut corners, Celest. Mech. Dyn. Astron., 59(3), 253-278.

Won, I.J. \& Bevis, M., 1987. Computing the gravitational and magnetic anomalies due to a polygon: Algorithms and fortran subroutines, Geophysics, 52(2), 232-238.

Zienkiewicz, O.C., Emson, C. \& Bettess, P., 1983. A novel boundary infinite element, Int. J. Numer. Methods Eng., 19(3), 393-404.

Zienkiewicz, O.C., Taylor, R.L. \& Zhu, J.Z., 2005. The Finite Element Method: Its Basis and Fundamentals, 6th edn, Elsevier ButterworthHeinemann.

\section{APPENDIX: ANALYTICAL SOLUTIONS}

In this section, we list all analytical solutions used in our benchmarks. In what follows, depth $z$ is positive downward.

\section{A1 Rectangular prism}

The analytical solution for the gravity anomaly due to a buried rectangular prism with a density anomaly $\delta \rho$ is given by (Plouff 1976 ):

$$
\begin{aligned}
\delta g_{z}(x, y, z) & =G \delta \rho \sum_{i}^{2} \sum_{j}^{2} \sum_{k}^{2} \mu_{i j k}\left[\delta z_{k} \tan ^{-1}\left(\frac{\delta x_{i} \delta y_{j}}{\delta z_{k} R_{i j k}}\right)\right. \\
& \left.-\delta x_{i} \ln \left(R_{i j k}+\delta y_{j}\right)-\delta y_{j} \ln \left(R_{i j k}+\delta x_{i}\right)\right] .
\end{aligned}
$$




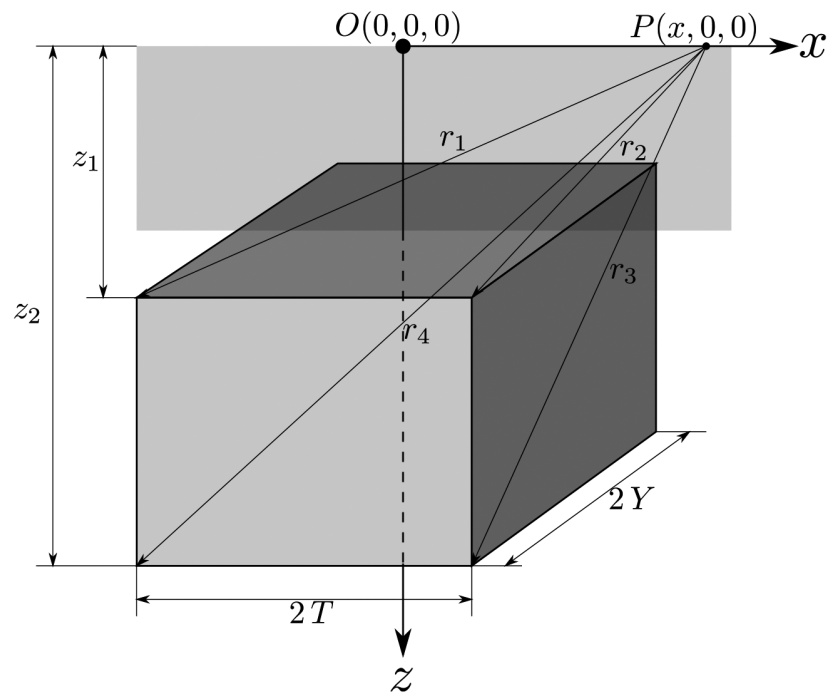

Figure A1. Geometry for the analytical gravity anomaly solution for a prism with a parabolic density distribution.

Here $\delta x_{i}=\left(x_{i}-x\right), \delta y_{j}=\left(y_{j}-y\right)$, and $\delta z_{k}=\left(z_{k}-z\right)$, where $x_{i}, y_{j}$, and $z_{k}$ denote the coordinates of the corners of the prism, $i, j, k=1,2$ and $R_{i j k}=\left(\delta x_{i}^{2}+\delta y_{j}^{2}+\delta z_{k}^{2}\right)^{1 / 2}$,

and

$\mu_{i j k}=(-1)^{i}(-1)^{j}(-1)^{k}$

\section{A2 Sphere}

The analytical solution for the gravity anomaly due to a sphere of radius $r$ with a density anomaly $\delta \rho$ buried at a depth of $z_{s}$ is (Turcotte $\&$ Schubert 2014)

$\delta g_{z}(x, 0,0)=\frac{4 \pi G r^{3} \delta \rho}{3} \frac{z_{s}}{\left(x^{2}+z_{s}^{2}\right)^{\frac{3}{2}}}$.

\section{A3 Multiple spheres}

The gravity anomaly induced by $n_{\mathrm{s}}$ spheres of radii $r_{s}$ and density anomalies $\delta \rho_{s}$ buried at depths $z_{s}$ is obtained by superposing the gravity anomalies of each individual sphere:

$\delta g_{z}(x, 0, z)=\frac{4 \pi G}{3} \sum_{s=1}^{n_{\mathrm{s}}} r_{s}^{3} \delta \rho_{s} \frac{z_{s}}{\left[\left(x-x_{s}\right)^{2}+\left(z-z_{s}\right)^{2}\right]^{\frac{3}{2}}}$.

\section{A4 Prism with a parabolic density distribution}

The gravity anomaly induced by a prism (Fig. A1) with a parabolic density anomaly distribution (eq. 31 ) is given by Chakravarthi et al. 
(2002). The expression in the original and subsequent references has typos. The correct form is

$$
\begin{aligned}
& \delta g_{z}(x, 0,0)=2 G \delta \rho_{0}^{3}\left\{\frac{\alpha Y(x+T)\left[2 \delta \rho_{0}^{2}+\alpha^{2}\left(Y^{2}+(x+T)^{2}\right)\right]}{L_{1}\left(Y^{2} \alpha^{2}+\delta \rho_{0}^{2}\right)\left[\delta \rho_{0}^{2}+\alpha^{2}(x+T)^{2}\right]} \ln \frac{L_{3}\left(\alpha r_{4} L_{1}+L_{1}^{2}-\delta \rho_{0} L_{4}\right)}{L_{4}\left(\alpha r_{1} L_{1}+L_{1}^{2}-\delta \rho_{0} L_{3}\right)}\right. \\
& -\frac{\alpha Y(x-T)\left[2 \delta \rho_{0}^{2}+\alpha^{2}\left(Y^{2}+(x-T)^{2}\right)\right]}{\left.L_{2}\left(Y^{2} \alpha^{2}+\delta \rho_{0}^{2}\right)\left[\delta \rho_{0}^{2}+\alpha^{2}(x-T)^{2}\right)\right]} \ln \frac{L_{3}\left(\alpha r_{3} L_{2}+L_{2}^{2}-\delta \rho_{0} L_{4}\right)}{L_{4}\left(\alpha r_{2} L_{2}+L_{2}^{2}-\delta \rho_{0} L_{3}\right)} \\
& \left.-\frac{\delta \rho_{0}}{\alpha\left(\delta \rho_{0}^{2}+Y^{2} \alpha^{2}\right.}\right)\left[\tan ^{-1} \frac{Y r_{4}}{z_{2}(x+T)}-\tan ^{-1} \frac{Y r_{1}}{z_{1}(x+T)}\right] \\
& \left.+\frac{\delta \rho_{0}}{\alpha\left(\delta \rho_{0}^{2}+Y^{2} \alpha^{2}\right.}\right)\left[\tan ^{-1} \frac{Y r_{3}}{z_{2}(x-T)}-\tan ^{-1} \frac{Y r_{2}}{z_{1}(x-T)}\right] \\
& -\frac{\delta \rho_{0}}{\alpha\left[\left(\delta \rho_{0}^{2}+(x+T)^{2} \alpha^{2}\right]\right.}\left[\tan ^{-1} \frac{(x+T) r_{4}}{z_{2} Y}-\tan ^{-1} \frac{(x+T) r_{1}}{z_{1} Y}\right] \\
& +\frac{\delta \rho_{0}}{\alpha\left[\delta \rho_{0}^{2}+(x-T)^{2} \alpha^{2}\right]}\left[\tan ^{-1} \frac{(x-T) r_{3}}{z_{2} Y}-\tan ^{-1} \frac{(x-T) r_{2}}{z_{1} Y}\right] \\
& +\frac{Y}{2\left(\delta \rho_{0}^{2}+Y^{2} \alpha^{2}\right)} \ln \frac{\left[(x+T)-r_{4}\right]\left[(x+T)+r_{1}\right]}{\left[(x+T)+r_{4}\right]\left[(x+T)-r_{1}\right]} \\
& -\frac{Y}{2\left(\delta \rho_{0}^{2}+Y^{2} \alpha^{2}\right)} \ln \frac{\left[(x-T)-r_{3}\right]\left[(x-T)+r_{2}\right)}{\left[(x-T)+r_{3}\right]\left[(x-T)-r_{2}\right]} \\
& -\frac{x-T}{2\left[\delta \rho_{0}^{2}+(x-T)^{2} \alpha^{2}\right]} \ln \frac{\left(Y-r_{3}\right)\left(Y+r_{2}\right)}{\left(Y+r_{3}\right)\left(Y-r_{2}\right)} \\
& +\frac{x+T}{2\left[\delta \rho_{0}^{2}+(x+T)^{2} \alpha^{2}\right]} \ln \frac{\left(Y-r_{4}\right)\left(Y+r_{1}\right)}{\left(Y+r_{4}\right)\left(Y-r_{1}\right)} \\
& +\frac{1}{\alpha\left(\delta \rho_{0}-\alpha z_{2}\right)}\left[\tan ^{-1} \frac{Y(x+T)}{z_{2} r_{4}}-\tan ^{-1} \frac{Y(x-T)}{z_{2} r_{3}}\right] \\
& \left.-\frac{1}{\alpha\left(\delta \rho_{0}-\alpha z_{1}\right)}\left[\tan ^{-1} \frac{Y(x+T)}{z_{1} r_{1}}-\tan ^{-1} \frac{Y(x-T)}{z_{1} r_{2}}\right]\right\} \text {, }
\end{aligned}
$$

where,

$$
\begin{aligned}
L_{1} & =\left[\left((x+T)^{2}+Y^{2}\right) \alpha^{2}+\delta \rho_{0}^{2}\right]^{\frac{1}{2}}, \\
L_{2} & =\left[\left((x-T)^{2}+Y^{2}\right) \alpha^{2}+\delta \rho_{0}^{2}\right]^{\frac{1}{2}}, \\
L_{3} & =\delta \rho_{0}-\alpha z_{1}, \\
L_{4} & =\delta \rho_{0}-\alpha z_{2},
\end{aligned}
$$

and

$$
\begin{aligned}
& r_{1}=\left[(x+T)^{2}+Y^{2}+z_{1}^{2}\right]^{\frac{1}{2}}, \\
& r_{2}=\left[(x-T)^{2}+Y^{2}+z_{1}^{2}\right]^{\frac{1}{2}}, \\
& r_{3}=\left[(x-T)^{2}+Y^{2}+z_{2}^{2}\right]^{\frac{1}{2}}, \\
& r_{4}=\left[(x+T)^{2}+Y^{2}+z_{2}^{2}\right]^{\frac{1}{2}} .
\end{aligned}
$$

